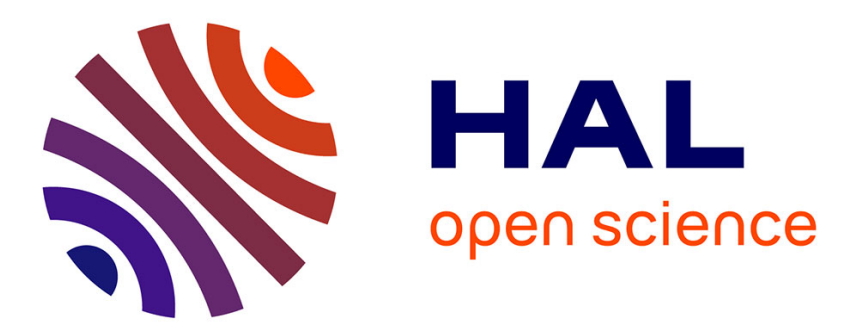

\title{
High frequency viscoelastic measurements of fluids based on microcantilever sensing: New modeling and experimental issues
}

Etienne Lemaire, Benjamin Caillard, Mohamed Youssry, Isabelle Dufour

\section{- To cite this version:}

Etienne Lemaire, Benjamin Caillard, Mohamed Youssry, Isabelle Dufour. High frequency viscoelastic measurements of fluids based on microcantilever sensing: New modeling and experimental issues. Sensors and Actuators A: Physical, 2013, 201, pp.230-240. 10.1016/j.sna.2013.07.022 . hal-00854141

\author{
HAL Id: hal-00854141 \\ https://hal.science/hal-00854141
}

Submitted on 13 Nov 2014

HAL is a multi-disciplinary open access archive for the deposit and dissemination of scientific research documents, whether they are published or not. The documents may come from teaching and research institutions in France or abroad, or from public or private research centers.
L'archive ouverte pluridisciplinaire HAL, est destinée au dépôt et à la diffusion de documents scientifiques de niveau recherche, publiés ou non, émanant des établissements d'enseignement et de recherche français ou étrangers, des laboratoires publics ou privés. 
pre-print SNA, 2013, EL, BC, MY, ID

\title{
High-frequency viscoelastic measurements of fluids based on microcantilever sensing: New modeling and experimental issues
}

\author{
Etienne Lemaire, Benjamin Caillard, Mohamed Youssry, Isabelle Dufour \\ Univ. Bordeaux, IMS, UMR 5218, F-33400 Talence, France \\ Email: etienne.lemaire@u-bordeaux1.fr
}

\begin{abstract}
In general, microrheology is carried out using active or passive particle-tracking techniques. In the present paper, a novel technique based on the out-of-plane bending vibrations of a microcantilever beam immersed into a liquid is proposed for microrheological property measurement. We propose to analytically link the damped beam motion with the rheological properties of the fluid in order to establish a dynamic rheogram which spans at least one decade of the kiloHertz frequency domain. The latest improvements in terms of both analytical modeling and experimental set-up are detailed, along with a complete explanation of the calculation method. Four rheograms of Newtonian and nonNewtonian liquids obtained from the frequency response of three immersed cantilevers of different geometries are presented.
\end{abstract}

Keywords: microcantilever, sensor, viscoelasticity, modeling, measurements, microrheology

\section{INTRODUCTION}

Exploring in-situ microrheology is of fundamental interest and is widely used in many applications, such as viscoelastic monitoring of screen-printing ink and small-volume texture monitoring of commercial foams or gels. This paper presents a method for the improvement of viscoelastic property extraction for complex fluids using a microcantilever as a dynamic rheometer oscillating in the kiloHertz domain [1]. This frequency range is difficult to explore with classical dynamic rheometers due to inertial limitations. MEMS rheometers [2,3] (as well as other mechanical resonators [4-6]) are good candidates as high-frequency rheometers (for determining elastic, $G^{\prime}$, and viscous, $G^{\prime \prime}$, shear moduli vs. frequency) by characterizing complex fluids at the microscale by using very small amounts of liquid. In this context, dynamic rheology is performed by exploring the use of small-scale devices and short characteristic times; this yields viscoelastic property measurements on downscaled specimen sizes not obtainable with traditional instruments, thereby forming the basis of "microrheology".

The concept of microrheology dates from 1922, when Freundlich and Seifriz used magnetic particles to study the elasticity of gelatin gels [7]. Over the last two decades, rapid developments have occurred in this field [8-11]. Renewed interest in microrheology has been increased by virtue of the technological innovations in colloidal engineering, light scattering, position sensitive detection and video microscopy. Currently there are two main categories of microrheology: active microrheology which involves the active manipulation of the probe particle by external forces (e.g., 
optical and magnetic tweezers and atomic force microscopy (AFM)) and passive microrheology which relies on the Brownian motion (thermal fluctuation) of the probe particles (e.g. video (VPT) and laser (LPT) particle tracking). Diffusing wave spectroscopy (DWS) is also a common microrheological technique widely used to characterize complex mixtures. As with other optical methods, DWS is based on the mean square displacement measurement which characterizes Brownian motion and permits the calculation of an equivalent microscopic and local shear modulus.

Microcantilevers vibrating in fluids are strongly influenced by the surrounding fluid properties [12]; thus, the out-of-plane transverse deflection response may be mathematically linked to the rheological properties of the surrounding liquid [13]. Based on previous work [14,15], we present here a considerably enhanced analytical model in order to (1) identify highly sensitive parameters in the determination method; (2) eliminate a misleading simplifying hypothesis in the solution; and (3) produce accurate rheograms in the case of non-Newtonian fluids. We also discuss the accuracy of the proposed method in order to examine the validity of the high-frequency rheograms produced and to demonstrate proof of concept. Using this new modeling approach, microcantilevers can probe the high-frequency viscoelastic behavior of fluids, especially the viscous characteristics, over a large frequency bandwidth around the resonant frequency. One of the main advantages of our technique is a faster response time than macrorheometers or other microrheological techniques.

\section{MODELING OF MICROCANTILEVER BEHAVIOR IN COMPLEX FLUIDS}

The Euler-Bernoulli differential equation (Eq. 1) governing the out-of-plane bending vibration of a microcantilever in a liquid medium is used to model the sensing situation:

$$
E I \frac{\partial^{4} w(x, \omega)}{\partial x^{4}}+j \omega g_{1}(x, \omega) w(x, \omega)-\omega^{2} g_{2}(x, \omega)+m_{L} w(x, \omega)=F_{e x t}(\omega) \delta(x-L)
$$

where $E$ is the Young Modulus, $I$ is the second moment of area of the rectangular cross section, $w(x, \omega)$ is the vibration amplitude at position $x$ along the length axis, depending on the radial frequency $\omega$ of the vibration, $L$ is the beam length and the position of the free-end of the cantilever, $m_{L}$ is the cantilever mass per unit length, $F_{\text {ext }}(\omega)$ is the applied force amplitude at the cantilever free-end, $\delta$ is the Dirac function and $g_{1}$ and $g_{2}$ are, respectively, functions of the dissipative and inertial part of the hydrodynamic force per unit length, $F_{\text {hydro }}$, exerted by the fluid on the cantilever.

Assuming that $g_{1}$ and $g_{2}$ are independent of $x$ [16], an exact analytical solution of Eq. 1 exists for the free-end cantilever deflection, $w(L, \omega)$. This solution gives a frequency-dependent response partially influenced by the rheological properties of the fluid:

$$
w(L, \omega)=\frac{3 F_{e x t}}{k_{0} A^{3} L^{3}} \frac{\sinh (A L) \cos (A L)-\cosh (A L) \sin (A L)}{1+\cosh (A L) \cos (A L)}
$$

where

$$
A^{4}=\frac{\omega^{2}}{E I} m_{L}+g_{2}(\omega)-j g_{1}(\omega) / \omega
$$

and $k_{0}$ is the cantilever stiffness. In Eqs. 2-3, the hydrodynamic properties of the surrounding fluid are contained in the terms $g_{1}$ and $g_{2}$ that are related to the imaginary and real parts of $F_{\text {hydro }}$. However, Eqs. 2-3 do not allow the inverse problem to be solved, meaning that $g_{1}$ and $g_{2}$ cannot be 
expressed explicitly as functions of the different parameters and measurement data $(\omega, w(L, \omega), E, I$, $\left.L, k_{0}, F_{\text {ext }}, m_{L}\right)$. Nevertheless, these analytical equations may be used to fit the experimental mechanical spectra of microcantilevers measured in known fluids. To do so, final rheological properties must be linked to $g_{1}$ and $g_{2}$, respectively, depending on the viscous and inertial parts of the hydrodynamic force:

$$
F_{\text {hydro }}=\omega^{2} g_{2}(\omega) w(x, \omega)-j \omega g_{1}(\omega) w(x, \omega)
$$

The differential equation solution supposes that the viscosity $\eta$ (hidden in the terms $g_{l}$ and $g_{2}$ ) is a real number. Viscoelastic characterization probed by microcantilevers is based on the assumption that the fluid viscosity can have both a real and an imaginary part. Consequently, the constant real term $\eta$ corresponding to viscosity is in this case replaced by a complex number $\eta^{*}$ accounting for both the viscous and elastic behavior of viscoelastic fluids. (This is classic within the context of rheological modeling $[17,18]$.) This notation is equivalent to considering a complex shear modulus $G^{*}$ for a viscoelastic fluid instead of an imaginary shear modulus. (See details in APPENDIX A). Moreover, a fluid is rheologically defined by a mass density $\rho_{f}$, and values $G$ ' and $G$ " which are, respectively, the elastic (real) part and the viscous (imaginary) part of the complex shear modulus $G^{*}$ at each frequency. A Newtonian fluid such as water has no elasticity $\left(G^{\prime}=0 \mathrm{~Pa}\right)$ and a viscosity with a frequency-independent real part $\left(\eta=10^{-3} \mathrm{~Pa} . \mathrm{s}=1 \mathrm{cP}\right.$, in the case of water). This defines a viscous modulus $G$ " that linearly increases with frequency $(G$ " $=\eta \omega)$. A fluid is considered as complex if it does not satisfy those two conditions.

In the case of thin rectangular microcantilevers, an exact equation of the hydrodynamic force has been developed, first by Sader [16] and then fitted by Maali et al. [19] in order to express separately the real and imaginary parts of the hydrodynamic force in terms of four constant coefficients $\left(a_{1}, a_{2}, b_{1}, b_{2}\right)$ whose values are dependent on the Reynolds number range. The accuracy of the fitting expressions are very good, provided that the appropriate Reynolds number range restriction has been verified. Based on those works, analytical equations of $g_{1}$ and $g_{2}$ as functions of the shear modulus were established [20]. Those expressions are recalled in Eqs. 5-7 (due to physical considerations detailed in APPENDIX A, $a_{1}=1$ and $a_{2}=b_{1}$ ):

$$
\begin{gathered}
g_{1}=D G^{\prime \prime}+B \sqrt{\sqrt{G^{\prime 2}+G^{\prime 2}}-G^{\prime}} \\
g_{2}=\frac{C+D G^{\prime}+B \sqrt{\sqrt{G^{\prime 2}+G^{\prime 2}}+G^{\prime}}}{\omega}
\end{gathered}
$$

where

$$
B=\frac{\pi b_{1}}{2 \sqrt{2}} b \sqrt{\rho_{f}} \quad C=\frac{\pi}{4} \rho_{f} b^{2} \omega \quad D=\frac{\pi b_{2}}{2 \omega}
$$

and $b$ is the cantilever width.

Using Eqs. 2-7 and considering a fluid with a known rheological behavior and a microcantilever with known geometry and material, it is possible to calculate the free-end deflection of the microstructure at each frequency and to compare it with experimental measurements. This also means that we are able to simulate experimental data. 


\section{INVERSE PROBLEM: METHOD FOR DETERMINATION OF RHEOLOGICAL FLUID PROPERTIES}

\section{A. From $g_{1}, g_{2}$ to $G^{\prime}, G^{\prime \prime}$}

With the expression of the hydrodynamic force from Sader [16] it is impossible to dissociate analytically the real part from the imaginary part, whereas it is possible with the approximation developed by Maali et al.[19]. Then, based on Eqs. 5-7, the elastic and viscous shear moduli can be expressed. (Details are presented in APPENDIX B.) The analytical expressions are

$$
\begin{gathered}
G^{\prime \prime}=\frac{g_{1}}{D}-\frac{B}{D \sqrt{2 D}} \sqrt{\sqrt{\left(\frac{B^{2}}{D}+2 \omega g_{2}-C\right)^{2}+4 g_{1}^{2}}-\frac{B^{2}}{D}-2 \omega g_{2}-C} \\
G^{\prime}=\frac{1}{D}\left(\omega g_{2}-C-\frac{B^{2} G^{\prime \prime}}{g_{1}-D G^{\prime \prime}}\right)
\end{gathered}
$$

The calculation of $G$ ' and $G$ " for any radial frequency $\omega$ can then be performed provided that $g_{1}$ and $g_{2}$ can be estimated with enough accuracy from the spectrum measurements. The calculation of the terms $g_{1}$ and $g_{2}$ as a function of both the amplitude and phase of the cantilever free-end deflection measurements is the purpose of the next section.

\section{B. Improvement of the inverse problem: from deflection to $g_{1}, g_{2}$}

The calculation of $g_{1}$ and $g_{2}$ from the amplitude and phase of the cantilever free-end deflection has been previously done by Belmiloud et al. [13]. At that time, the authors have considered that the analytical solution (Eq. 2) of the differential equation (Eq. 1) can be approximated by a second order mechanical low-pass filter with the transfer function $H$ considered as follows:

$$
H(f)=\frac{w}{F_{\text {ext }}} \approx \frac{H_{0}}{1-\left(1+\frac{g_{2}(\omega)}{m_{L}}\right)\left(\frac{\omega}{\omega_{0, v a c}}\right)^{2}+j \frac{g_{1}(\omega) \omega}{m_{L} \omega_{0, v a c}^{2}}}
$$

$\omega_{0, v a c}$ is the cantilever's fundamental radial eigenfrequency in vacuum.

Eq. 10 is almost equivalent to the Taylor series development of Eq. 2 as a function of the nondimensional term $A L$. However, assuming the non-dimensional term $Z=(A L)^{4}$, it is also almost equivalent to the ratio of two polynomial functions of $Z$, with the numerator order equal to 0 and the denominator order equal to 2 ; this corresponds to taking only the first resonant mode into account. In order to improve the accuracy of the approximating function to Eq. 2, orders were added to the numerator and the denominator by expanding the Taylor series to higher orders. Examples of these results are presented in Fig. 1. As the Taylor series order is increased, the exact transfer function is approached more and more accurately. 


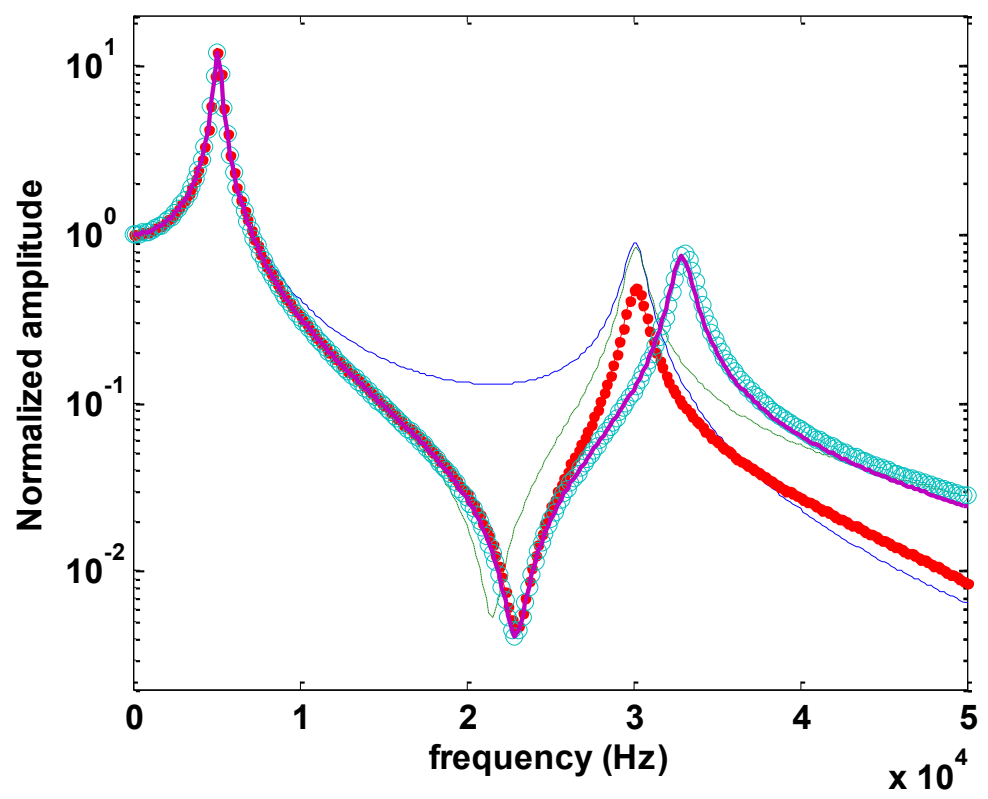

Fig. 1 - Approximation of the amplitude of the transfer function (Eq. 2) for a $1440 \times 285 \times 20 \mu \mathrm{m}^{3}$ silicon microcantilever with numerator and denominator orders respectively equal to 0 and 2 (blue), 1 and 2 (dashed green), 2 and 2 (dotted red), 3 and 3 (dotted light blue) which correspond to Taylor series of increasing orders. The reference curve (Eq. 2 is shown in purple)

An optimum has been searched for in order to obtain both high accuracy and a quite simple expression of the transfer function $H$ so that $Z$ (hence $A, g_{1}, g_{2}, G^{\prime}$ and $G$ ') can be expressed analytically as a function of $H$. The Taylor series development order has been fixed to obtain numerator and denominator orders of three. This process led to the following approximate equation, which will be used in this paper instead of Eq. 10:

$$
\frac{H}{H_{0}}=\frac{k_{0} w(L, \omega)}{F_{\text {exc }}}=\frac{k_{0}}{A^{3} E I} \frac{\sinh (A L) \cos (A L)-\cosh (A L) \sin (A L)}{1+\cosh (A L) \cos (A L)} \approx 3 \frac{\frac{Z^{3}}{2554051500}-\frac{Z^{2}}{311850}+\frac{Z}{315}-\frac{4}{3}}{-\frac{Z^{3}}{37424200}+\frac{Z^{2}}{1260}-\frac{Z}{3}+4}
$$

This is a quotient of third order polynomials in $Z$ with $H$ as a parameter. ( $H_{0}$ is the static value of $H$ ). Each one of the three solutions (the analytical expressions are detailed in APPENDIX C1) corresponds to a physical solution over different frequency ranges. As an illustration, Fig. 2 shows $g_{1}$ obtained with each of the solutions and with the solution of Eq. 10 for a specific complex fluid ${ }^{1}$ and a specific cantilever whose first- and second-mode resonant frequencies are equal to $f_{0}$ and $f_{1}$; solution 3 is valid over a larger frequency range, until $10 \mathrm{kHz}$ in the simulated case. Then, using the appropriate solution depending on the considered frequency range, the hydrodynamic behavior can be calculated up to $30 \mathrm{kHz}$, that is, up to $f_{1}$. Furthermore, results obtained with Eq. 11 are significantly more accurate than those obtained with Eq. 10, even at low frequency. Similar results are obtained for $g_{2}$ and presented in APPENDIX C1.

\footnotetext{
${ }^{1}$ The simulated complex fluid corresponds to data extracted from literature, fitted on Buchanan et al.'s results. The $G$ ' and $G$ "' are fitted from a $4 \mathrm{wt} \%$ CTAB solution, characterized in [21].
} 


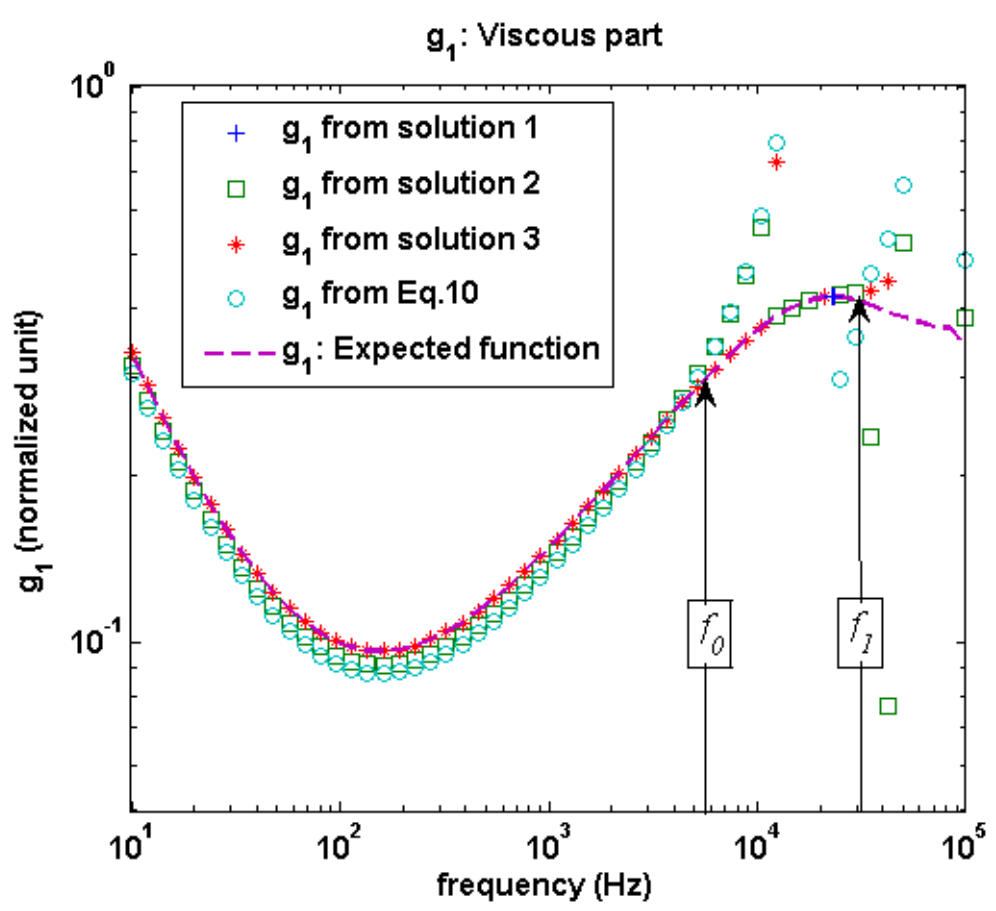

Fig. 2 - Viscous dissipative term $\left(g_{1}\right)$ respectively calculated from the three solutions $\left(Z_{1}:+, Z_{2}: \square, Z_{3}: *\right)$ of Eq. 11, from the solution of Eq. 10 (o) and expected (-) for a $1440 \times 285 \times 20 \mu \mathrm{m}^{3}$ silicon microcantilever immersed into a simulated complex fluid ${ }^{2}$.

By joining the different solutions $Z_{1}, Z_{2}$ and $Z_{3}$ of this upgraded Taylor approximation, the viscous $\left(g_{1}\right)$ and inertial $\left(g_{2}\right)$ parts of the hydrodynamic force are now estimated from the free-end cantilever deflection amplitude and phase with a better accuracy and over a larger frequency range than in previous works. By using Eqs. 8 and 9, it is now possible to analytically calculate the complex shear modulus as a function of the free-end cantilever deflection. This method leads to accurate rheograms of complex fluids over a large range of frequency from $0 \mathrm{~Hz}$ to the secondmode resonant frequency $f_{l}$, whereas it was previously limited to the first-mode resonant frequency $f_{0}$, and quite inaccurately.

Further improvements in the solution of Eq. 11 are presented in APPENDIX C2 that will be used in the following sections, thus ensuring high accuracy even at frequencies slightly above $f_{l}$.

\section{From simulated experimental data to rheograms: discussion on accuracy}

\section{Influence of the approximation made during the inverse problem}

In this part, experimental data are generated by simulation from input rheological properties of fluids and geometrical and material properties of cantilevers (see part I), and rheograms are produced with Eqs. 8- 10 (previous work) or 11 (this work). The produced rheograms differ from the original ones only due to the approximation assumed with the inverse problem. Nevertheless, the error introduced by this approximation can be estimated.

\footnotetext{
${ }^{2}$ The simulated complex fluid corresponds to data extract from literature, fitted on Buchanan et al.'s results. The $G$ ' and $G$ "' are fitted from a $4 \mathrm{wt} \%$ CTAB solution, characterized in [21].
} 
In Figs. 3 and 4, the geometry of the silicon microcantilever is $1440 \times 285 \times 20 \mu \mathrm{m}^{3}$, and the fluid used is water (with $G^{\prime \prime}=\eta \omega$, where $\eta=10^{-3} \mathrm{~Pa} . s$, and $\mathrm{G}^{\prime}=10^{-11} \mathrm{~Pa}$ instead of 0 ); in Fig. 5, the microcantilever is the same, but the fluid is a complex fluid which corresponds to data extracted from the literature, fitted on Buchanan et al.'s results (the $G$ ' and $G$ "' are fitted from a $4 \mathrm{wt} \%$ CTAB solution, characterized in [21]).

The determination of $G$ " (Figs. 3 and 5) is accurate from $10 \mathrm{~Hz}$ to the second-mode resonant frequency $f_{1}$ for both fluids by using Eq. 11. In Fig. 3, it also clearly appears that using Eq. 10 leads to inaccuracy over the entire frequency range considered.

The determination of $G$ ' of the complex fluid is also accurate (Fig. 5), whereas it is more troublesome in the case of water (Fig. 4). The interpretation is that the approximation becomes an issue for very low values of $G^{\prime}$. The validity of the inverse model is demonstrated with simulation data for $G^{\prime}$ and $G$ "' higher than $1 P a$. More specifically, it means that even for Newtonian fluids, the calculated value of $G^{\prime}$ will be non-zero.

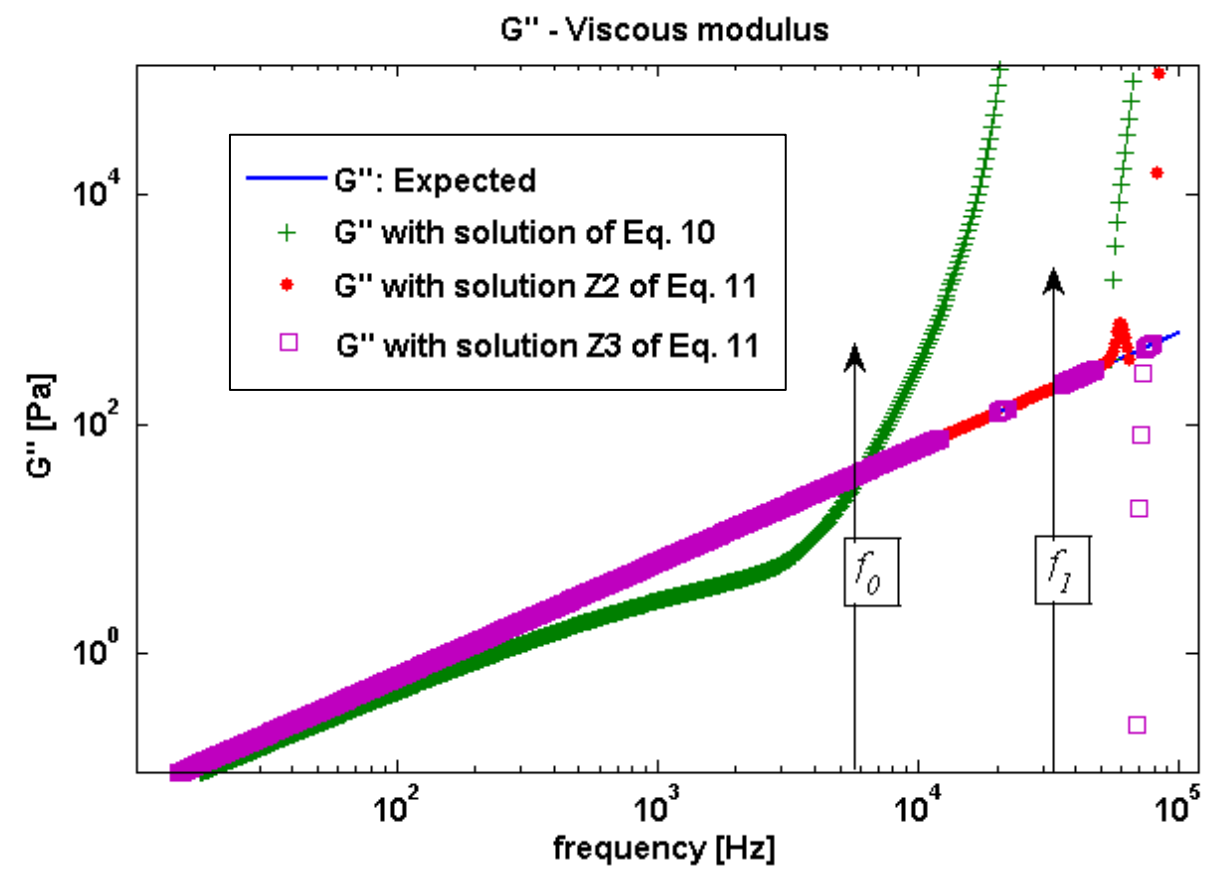

Fig. 3 - Viscous shear modulus $\left(G\right.$ ”) calculated from two of the solutions $\left(Z_{2}: \bullet, Z_{3}: \square\right)$ of Eq. 11, from the solution of Eq. $10(+)$ and expected (-) for a $1440 \times 285 \times 20 \mu \mathrm{m}^{3}$ silicon microcantilever immersed in water. 


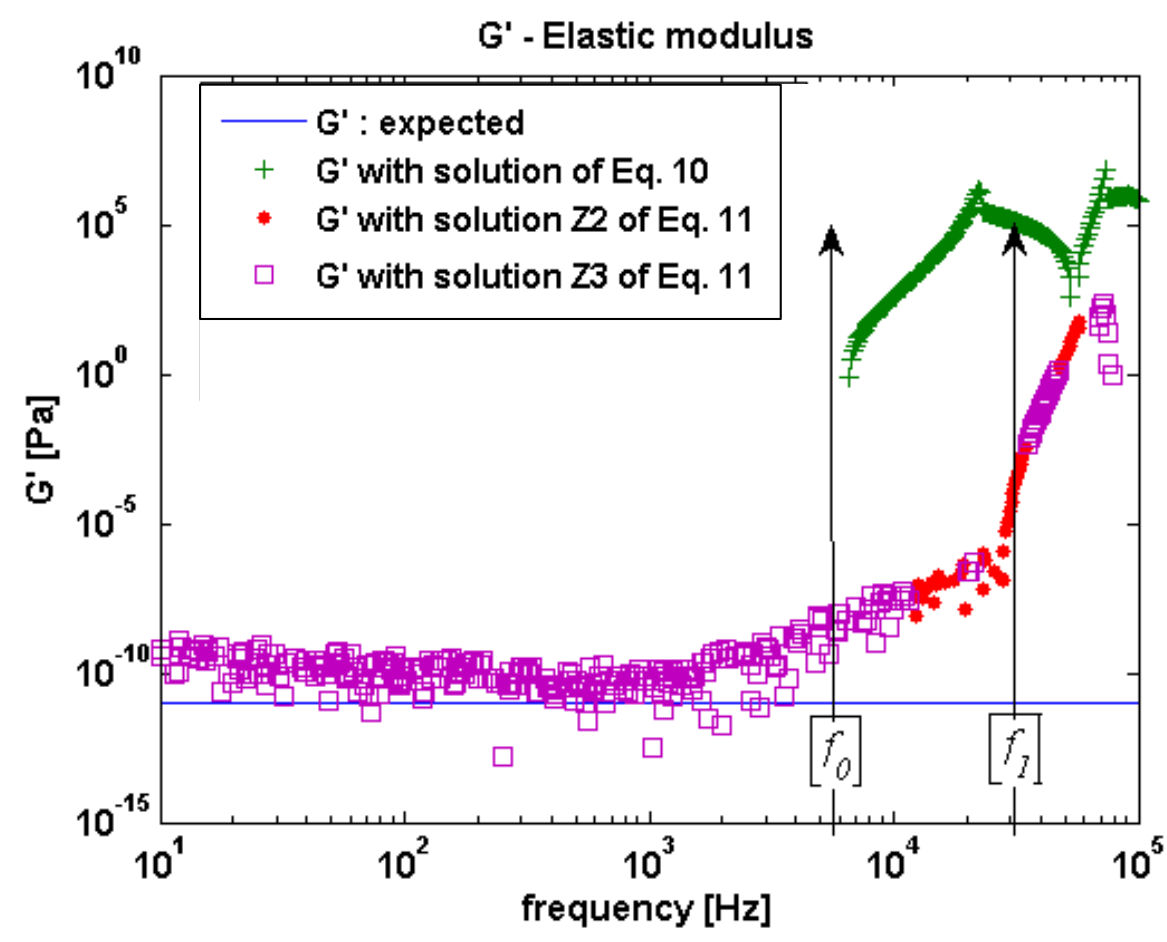

Fig. 4 - Elastic shear modulus $\left(G^{\prime}\right)$ calculated from two of the solutions $\left(Z_{2}: \bullet, Z_{3}: \square\right)$ of Eq. 11, from the solution of Eq. $10(+)$ and expected (-) for a $1440 \times 285 \times 20 \mu \mathrm{m}^{3}$ silicon microcantilever immersed in water $\left(G^{\prime}=0\right.$, simulated here with $\left.G^{\prime}=10^{-11} \mathrm{~Pa}\right)$.

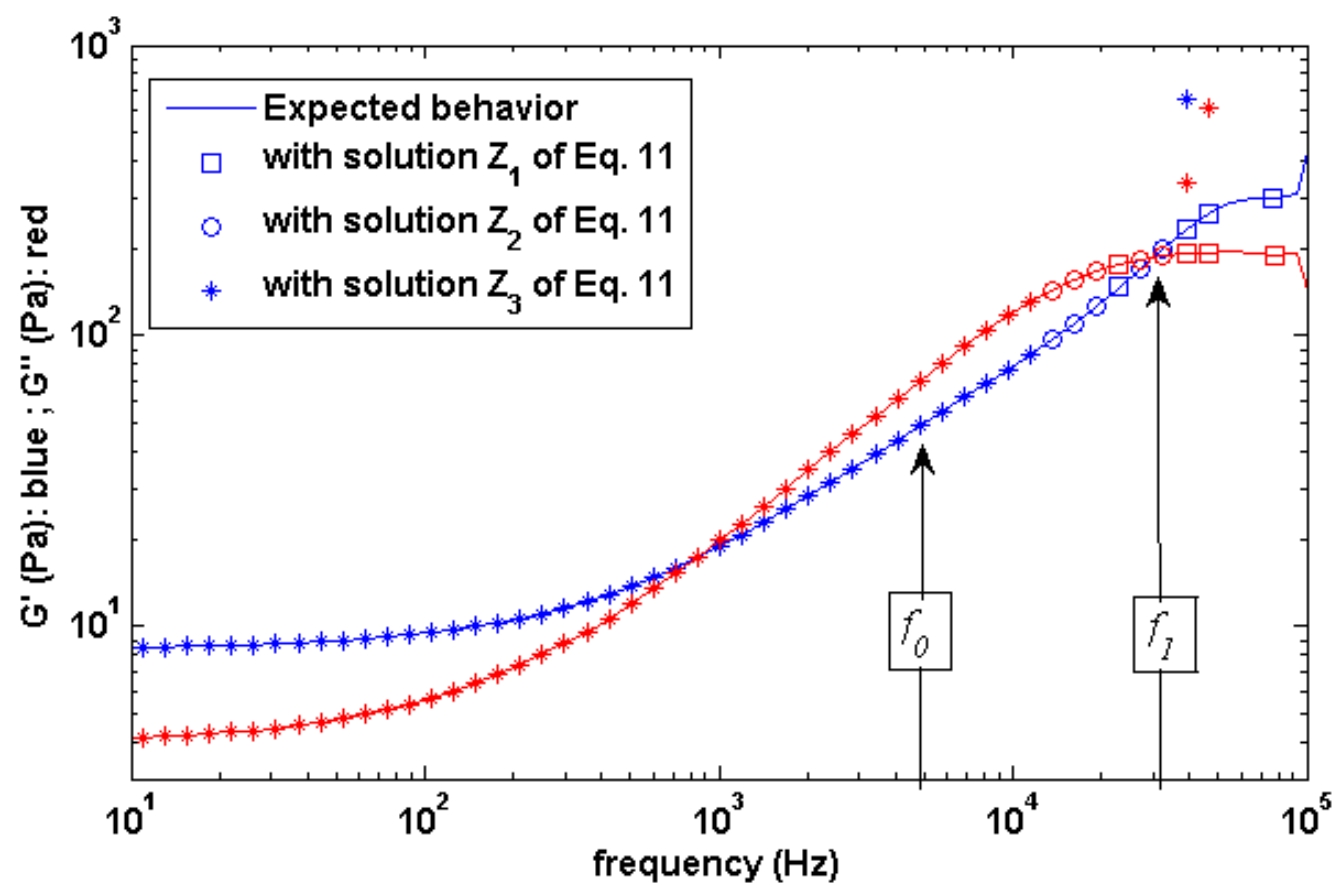

Fig. 5 - Elastic ( $G^{\prime}$ : blue) and viscous ( $G^{\prime \prime}$ : red) moduli calculated from the three solutions $Z_{1}, Z_{2}$ and $Z_{3}$ of Eq. 11, and the expected functions which have been used

to generate the simulation data (-) in the case of a $1440 \times 285 \times 20 \mu \mathrm{m}^{3}$ silicon microcantilever immersed in a complex fluid. 
Nevertheless this systematic error does not matter as it will be shown in part II.C.2 and in part III. Its influence on the accuracy of the produced rheograms is negligible when compared to the error related to model parameter uncertainties and to the experimental signal to noise ratio.

\section{Influence of model parameters}

As all of the geometrical and physical parameters are not known precisely, the results obtained with simulated experimental data do not validate the measurements results. For instance, in Fig. 6, the influence of an error on $\mathrm{H}_{0}$, corresponding to the static deflection of the cantilever normalized by the applied actuation voltage (the applied actuation force is proportional to the applied actuation voltage), is tested. Undoubtedly, the $G$ ' estimation is strongly dependent on the value of $H_{0}$. The estimation of the viscous modulus $(G$ ") is also dependent on this parameter in the same proportions.

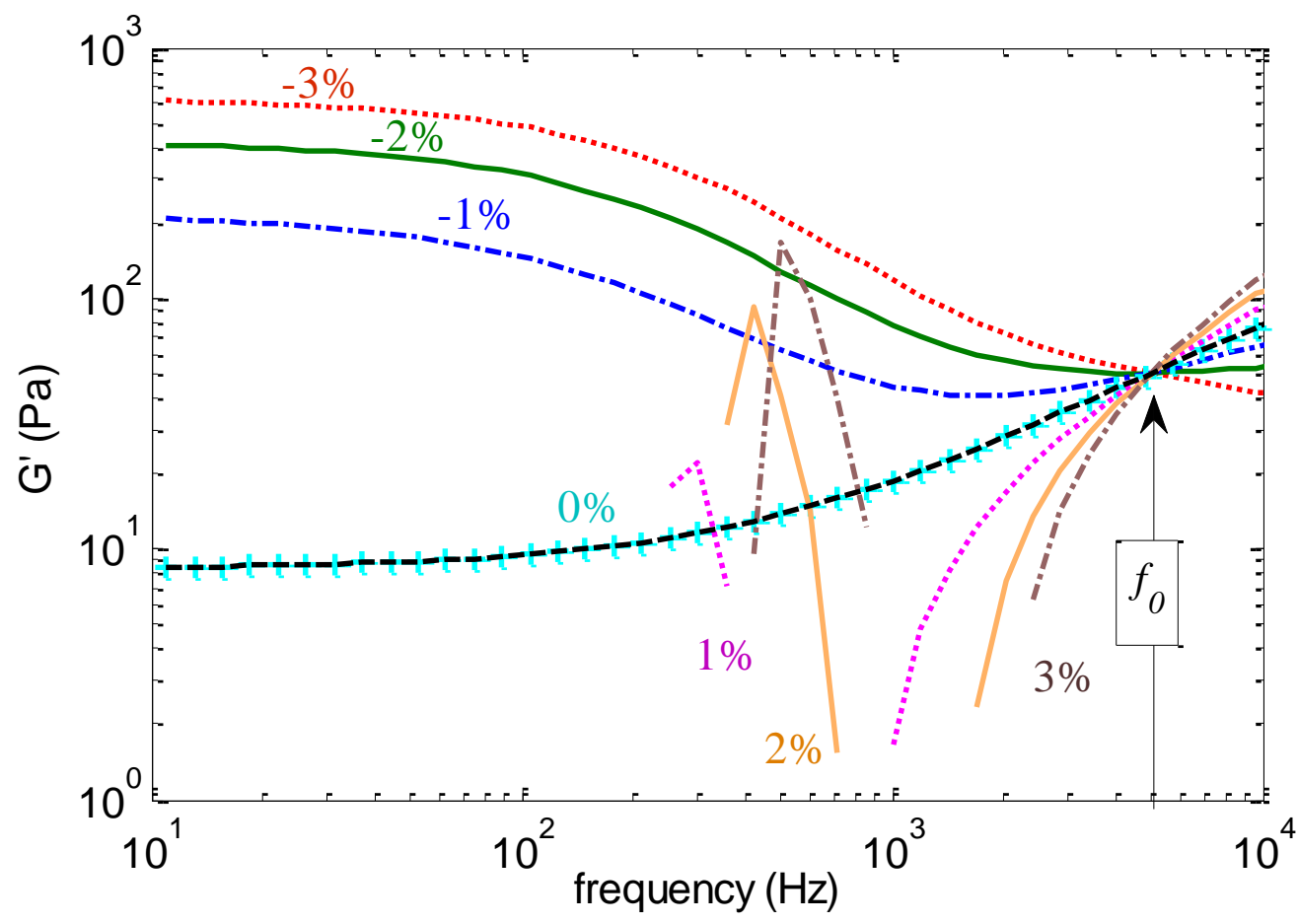

Fig. 6 - Elastic modulus $\left(G^{\prime}\right)$ calculated from the third solution $\left(Z_{3}\right)$ expressed in Eq. C11, with respectively $-3 \%$ (red dotted line), $-2 \%$ (green line), $-1 \%$ (blue dotted line), $0 \%$ (light blue cross), $1 \%$ (magenta dotted line), $2 \%$ (orange line), 3\% (brown dotted line) of error on $H_{0}$ and the expected function for a $1440 \times 285 \times 20 \mu \mathrm{m}^{3}$ silicon microcantilever immersed in a complex fluid (-).

In Table 1, a set of measured values of $H_{0}$ are presented for three cantilevers of different geometries. Those values strongly differ from one cantilever to another depending on manufacturing considerations, such as the position of the structure on the silicon wafer. In addition, the low frequency noise of the vibrometer used for the measurement of the microcantilever free-end deflection makes a direct measurement impossible and an optical profilometer or any optical measurement technique would not satisfy the required accuracy (in practice the applied actuation voltage is set to $0.1 \mathrm{~V}$, so according to Table 1 the absolute value of the deflection is at the nanoscale and a resolution of less than one percent is required). Therefore, it becomes difficult to measure those values experimentally with an accuracy better than $1 \%$. 
pre-print SNA, 2013, EL, BC, MY, ID

\begin{tabular}{|c|c|c|c|c|}
\hline $\begin{array}{l}\text { Cantilever } \\
\text { name }\end{array}$ & $\begin{array}{l}\text { Cantilever } \\
\text { geometry }\end{array}$ & $\begin{array}{c}H_{0, \min } \\
\left(\mathrm{m} . \mathrm{V}^{-1}\right)\end{array}$ & 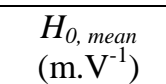 & $\begin{array}{c}H_{0, \max } \\
\left(\mathrm{m} \cdot \mathrm{V}^{-1}\right)\end{array}$ \\
\hline $\mathrm{LL}$ & $2810 \times 100 \times 20 \mu \mathrm{m}^{3}$ & $1.810^{-8}$ & $2.3310^{-7}$ & $3.010^{-7}$ \\
\hline LH & $1440 \times 285 \times 20 \mu \mathrm{m}^{3}$ & $1.010^{-8}$ & $2.95610^{-8}$ & $5.010^{-8}$ \\
\hline $\mathrm{A}$ & $500 \times 100 \times 20 \mu \mathrm{m}^{3}$ & $1.510^{-11}$ & $9.37710^{-10}$ & $1.310^{-9}$ \\
\hline
\end{tabular}

Table 1 - Measured values of $H_{0}$ in water for the three cantilevers of different geometries

A fitting and iterative method has been developed in order to determine precisely $H_{0}$ (the complete calculation method is not detailed in this paper).

Parametrical errors (like the one concerning $H_{0}$ ) remain a problem, especially because few reference measurements exist for the probed complex fluids at the investigated frequency range. In this context, it is difficult to calibrate those parameters or to correct the results with a simple selfcalibration. Nevertheless, Fig. 6 shows that whatever the residual parametrical error on $H_{0}$, the values of $G$ ' (and $G$ ") calculated at the resonant frequency is "exact". Consequently, in the experimental rheograms the more accurate measurements of $G$ ' and $G$ " are obtained at the first resonant frequency. At other frequencies, an error on $H_{0}$ or one of the other sensitive parameters (not detailed in this article) will strongly affect both the value and the slope of $G$ ' and $G$ " vs. frequency.

\section{DETERMINATION OF RHEOLOGICAL PROPERTIES OF FLUIDS USING MICROCANTILEVER MEASUREMENTS}

\section{A. Experimental set-up and procedure}

In order to characterize the viscosity of various fluids, silicon microcantilevers have been designed and fabricated [22]. Three different geometries have been used for the measurements (specimens 'LL', 'LH' and 'A'). They all have the same thickness (SOI wafer) but different lengths and widths, as presented in Table 1. An SEM image of a microcantilever can be seen in Fig. 7C, while the experimental set-up is summarized in Fig. 7A. The dynamic deflection (amplitude and phase) of the cantilevers has been measured optically using a laser vibrometer (MSA500, Polytec). The optical vibrometer is used in this paper because it provides accurate results, characterizing the dynamic behavior of the microstructure without any coupling or signal distortion issues, over a wide range of frequency. Silicon microcantilevers are actuated in the liquid medium (see Fig. 7B) by the electromagnetic Laplace force resulting from the interaction between an external magnetic field generated by a magnet and an electrical current generated by an external electrical voltage in a gold conductive path designed on the surface of the microcantilevers.

Four different liquids have been characterized over a large frequency range $\left(10^{3}-10^{5} \mathrm{~Hz}\right)$ with a controlled room temperature of $19^{\circ} \mathrm{C}$ : two Newtonian fluids, deionized water and a $20-\mathrm{cP}$ calibrated silicone oil (provided by Sigma Aldrich). In addition two complex viscoelastic fluids, mixtures of cetyltrimethylammonium bromide (CTAB) and polyacrilamide (PAM), were tested.

Two different solutions of PAM were prepared by adding $50 \mathrm{mM}$ and $100 \mathrm{mM}$ of PAM, respectively, to $10 \mathrm{mM}$ of $\mathrm{NaCl}$. CTAB was concentrated as follow: $100 \mathrm{mM}$ of surfactant added to $40 \mathrm{mM}$ of NaSal.

The same protocol has been used for the three cantilever geometries. First the cantilever deflection spectrum was measured in air (the eigenfrequency in vacuum needed for the rheogram calculation was deduced from the measurement in air). Then the cantilever resonant spectrum was acquired in deionized water. Finally the measurements in the complex mixture or in the silicone oil 
were obtained. Each presented experimental spectrum was obtained in less than one minute by averaging 100 spectra. In these conditions, for a given cantilever in a given fluid, repeatability was excellent provided that temperature does not change and that the concentration of the mixtures does not fluctuate due to, e.g., evaporation. This is guaranteed in our set-up by a room temperature control and by performing all the measurements in a short period of time after exposing a new sample to air. The measurements obtained with two identically designed cantilevers are slightly different due to small differences caused by the manufacturing process. This is the reason why a calibration procedure in water has been developed.

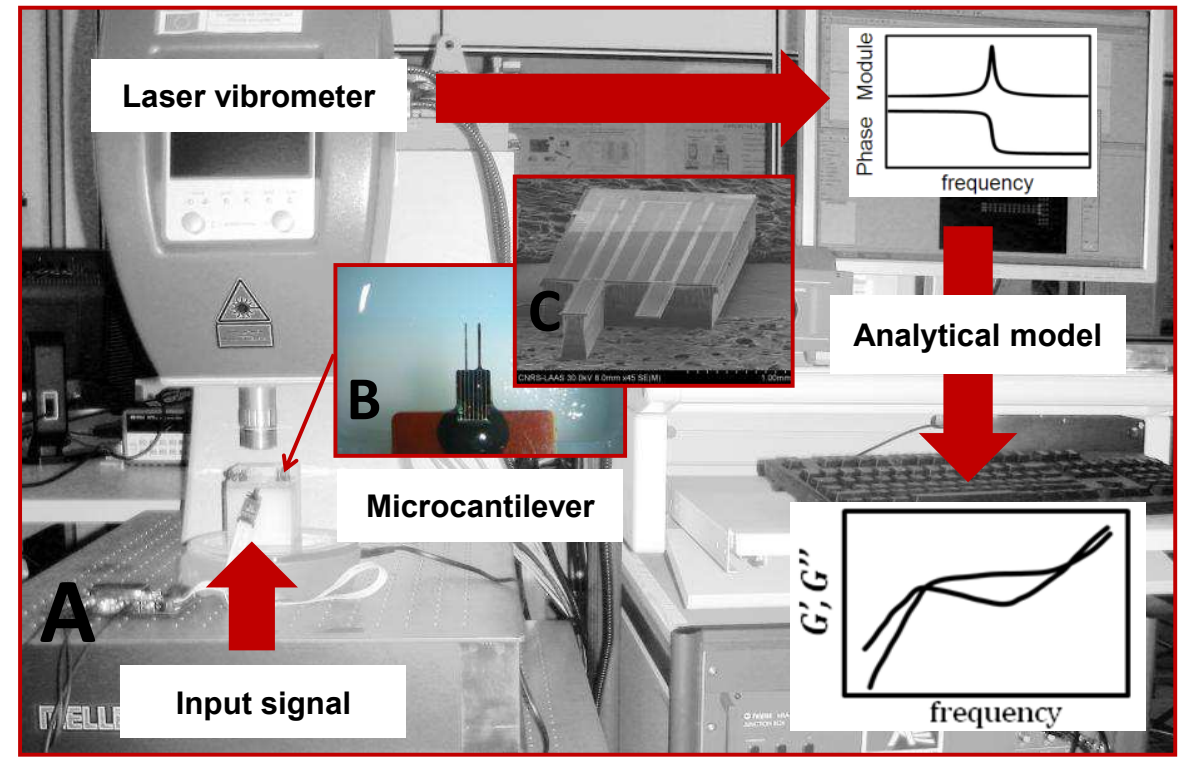

Fig. 7 - Experimental set-up illustration. Method summary (A); view of a microcantilever within liquid recipient (B); an SEM photo of the silicon-etched device $(\mathrm{C})$.

Different sets of cantilevers were used for each complex fluid. The silicone oil was also characterized using other cantilevers for cleaning considerations. (Oil leaves many traces on the cantilever surface that are difficult to clean in water-based solutions.)

\section{B. Newtonian Fluids}

\section{Calibration in water}

Even though very few reference measurements exist over the investigated frequency range, many rheologists (see acknowledgments) believe that there is no single physical reason why water should deviate from Newtonian behavior: to our knowledge, it is the best existing reference fluid (it could be suspected that some Newtonian calibration fluids composed of large molecules might partially behave as non-Newtonian fluids at high frequency when considered at very small scales, see Fig. 10). Therefore, measurements in deionized water should lead to a very small value of $G$ ' and a constant viscosity (versus frequency) close to $1 \mathrm{cP}$, depending only on temperature.

A calibration method based on these expected measurements in water is used to calibrate the model parameters identified as crucial for the accuracy of the experimental shear modulus determination. This method depends on the cantilever's geometric parameters $(h, b, L)$, the cantilever mass per unit length $\left(m_{L}\right)$ and $H_{0}$, and is based on minimizing the mismatch between the 
experimental and the simulated spectra obtained using the method developed in parts I and II, assuming specified variations in these five parameters of a few percent around their nominal values. The nominal values of $h, b$ and $L$ are taken directly from the cantilever mask design values. The nominal parameter $m_{L}$ is calculated using the cantilever mask design values and the actual cantilever density, taking into account that it is made of layers of different materials $\left(\mathrm{Si}, \mathrm{SiO}_{2}, \mathrm{Au}\right.$, Ti). The nominal value of $H_{0}$ comes from the above-mentioned fitting and iterative method and is not detailed in this paper.

Fig. 8 presents the three microcantilevers' deflection measurements when the devices are immersed in water and the results of the best simulation cases; as can be seen, the comparisons are excellent. The results of the calibration parameters corresponding to the three cantilevers (LL, LH and A) immersed in water are presented in Table 2. The variations of parameters are deemed to be physically reasonable.
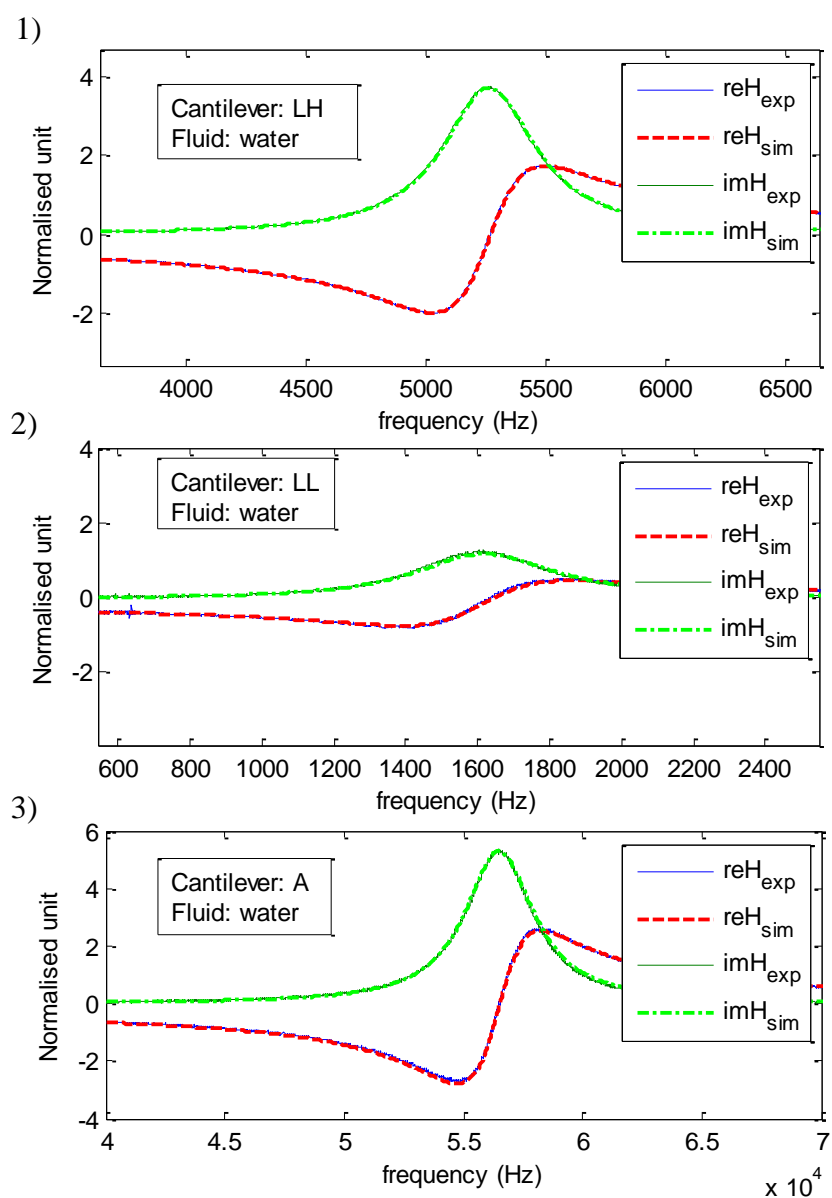

Fig. 8 - Real $(\mathrm{reH})$ and imaginary $(\mathrm{imH})$ parts of experimental and simulated freeend deflection complex modulus $(H)$ for the three geometries of cantilevers immersed in water: 1) LL; 2) LH; 3 ) A. 


\begin{tabular}{|c|c|c|c|c|c|}
\hline $\begin{array}{c}\text { Percentage } \\
\text { change on }\end{array}$ & $\boldsymbol{h}$ & $\boldsymbol{b}$ & $\boldsymbol{L}$ & $\boldsymbol{H}_{\boldsymbol{0}}$ & $\boldsymbol{m}_{\boldsymbol{L}}$ \\
\hline $\mathrm{LL}$ & 3.0 & 0 & 6.0 & 1.9 & 0 \\
\hline $\mathrm{LH}$ & 1.4 & 0 & $4.510^{-2}$ & -1.5 & 0.11 \\
\hline $\mathrm{A}$ & $3.710^{-1}$ & 0 & 0 & 5.2 & 0 \\
\hline
\end{tabular}

Table 2 -Model parameter variations around their nominal values obtained to optimally simulate the free-end cantilever deflection which corresponds to the one measured for each geometry (LL, LH or A) immersed in water.

The rheogram presented in Fig. 9 illustrates the Newtonian behavior of water obtained with the three cantilever spectrum measurements. Referring to the curve illustrating the influence of $H_{0}$ (cf. Fig. 6), both $G$ ' and $G$ "' are calculated with an error which modifies the slope of those rheograms; the same influence is observed for the other model parameters. This is particularly observable in the case of cantilever A. The fact discussed in the simulation description (section II-C) that we are not able to accurately measure the elastic shear modulus under $1 \mathrm{~Pa}$ is confirmed: the behavior of $G$ ' appears to be clearly inconsistent. However the behavior of $G$ " (and therefore the viscosity) is very close to the expected behavior in terms of the quantitative values and the continuity between the measurements made using different microcantilevers.

More than one decade separates both portions of the complex modulus; thus, $G$ ' can be considered negligible as compared to $G$ ". In the opinion of the authors, this is sufficient to confirm the Newtonian behavior of water.

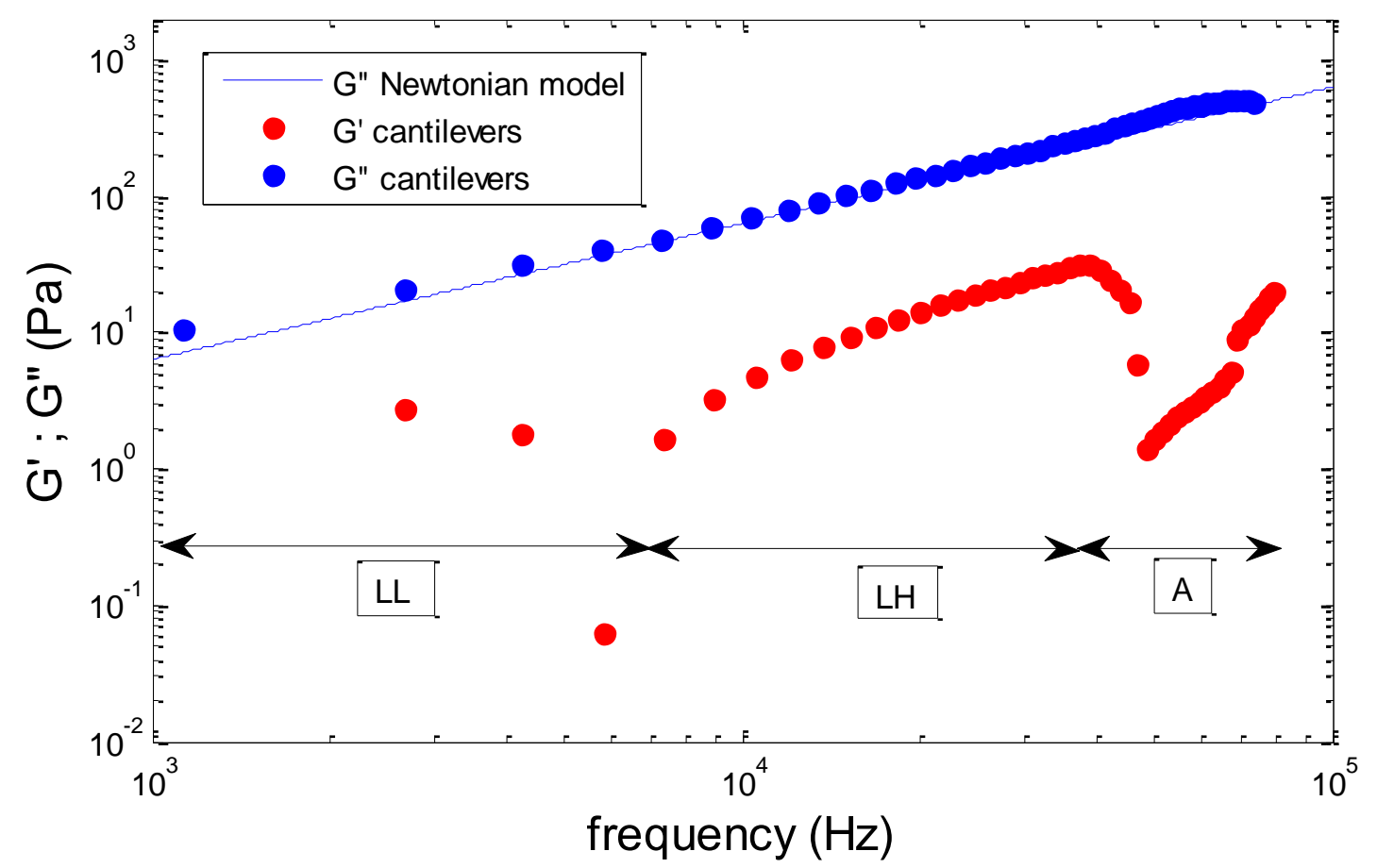

Fig. 9 - High-frequency rheogram of water measured with three microcantilevers (LL, LH and A) and spanning the frequency range $10^{3}-10^{5} \mathrm{~Hz}$ using the inverse model (Eqs. 8-9 and 11). 


\section{Rheogram of a 20 cP silicone oil}

The model parameter values obtained after calibration in water for each cantilever were kept unchanged during measurements achieved in a $20 \mathrm{cP}$ silicone oil. The corresponding obtained rheogram is presented in Fig. 10.

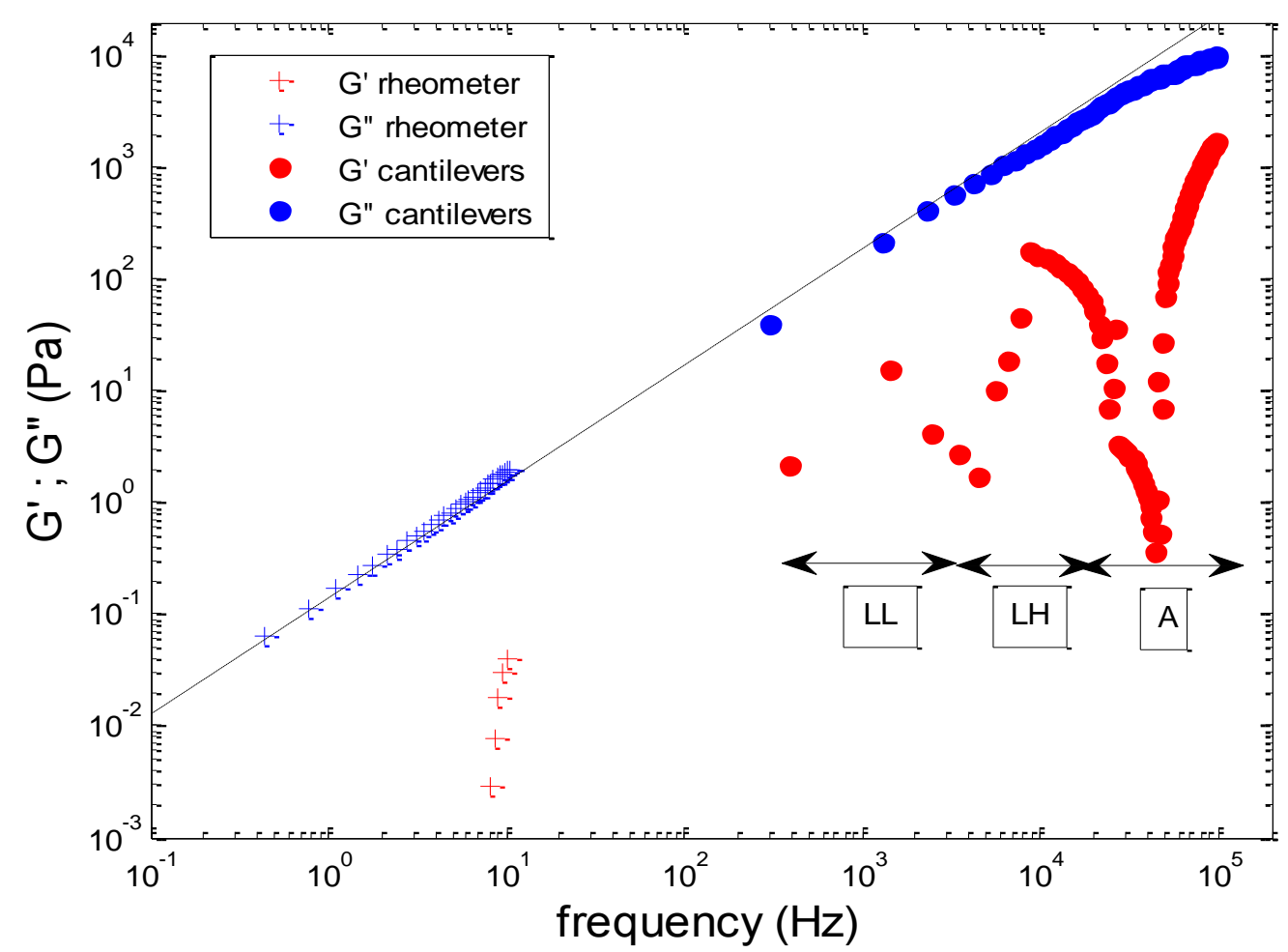

Fig. 10 -Rheogram of a 20-cP silicone oil measured with a classical cone-plate rheometer and three microcantilevers (LL, LH and A) spanning the frequency range $10^{3}-10^{5} \mathrm{~Hz}$ using the inverse model (Eqs. 8-9 and 11).

The behavior of $G$ " is as expected because there is continuity between the measurements obtained with the classical rheometer at low frequencies and the measurements made with the different cantilevers. The obtained viscosity value of $21.3 \mathrm{cP}$, is in agreement with the expected viscosity at $19^{\circ} \mathrm{C}$ for this silicone oil (measurement made with a cone/plate rheometer with a Peltier temperature control).

More than one decade of difference is systematically obtained between $G$ ' and the larger value $G$ ”, which is deemed to be large enough for indicating Newtonian behavior. This is particularly true at the resonant frequency, where the model parameter influence is the weakest: the value of $G$ ' is more than two decades lower than the value of $G$ ". According to the $G$ " high frequency slope, it can be suspected that the fluid become complex at very high frequency, i.e., in the megahertz range.

The results obtained with silicone oil and water using three microcantilever geometries (LL $\mathrm{LH}, \mathrm{A}$ ) demonstrate that the viscous modulus $G$ " determination between $10^{-1}-10^{4} \mathrm{~Pa}$ is possible in the extended range of frequencies $10^{3}-10^{5} \mathrm{~Hz}$.

\section{Complex Fluids}

Characterization of CTAB and PAM, prepared as mentioned above, are presented in Figs. 11 and 12, respectively. They are supposed to be complex fluids behaving according to linear 
viscoelasticity with our experimental set-up, a point that has been carefully checked in a previous work [23]. The limit to the linear regime depends on the amplitude of the applied pressure due to the cantilever motion and therefore on the amplitude of the driving voltage. In our case, the pressure that defines the limit between the linear and non-linear viscoelastic regimes, has been measured around $1 \mathrm{~Pa}$ at $10 \mathrm{kHz}$ for the CTAB mixture. This corresponds to approximately 800 $\mathrm{mV}$ for the LH cantilevers whereas all of the presented measurements have been performed at 100 $\mathrm{mV}$.

Fig. 11 presents our results for $G$ ' and $G$ "' of CTAB obtained at $19^{\circ} \mathrm{C}$ both with cantilevers and with a cone-plate rheometer as well as published [24] DWS reference measurements with the same mixture obtained at $22^{\circ} \mathrm{C}$. As expected, unlike Newtonian fluids, $G$ ' is no longer negligible. Except for the $G^{\prime}$ measurements obtained with the LL cantilevers $\left(G^{\prime}{ }_{L L}\right)$, all sets of measurements of $G$ ' and $G$ " clearly follow the same trends until $100 \mathrm{kHz}$, given the temperature difference. The inconsistent results obtained for $G^{\prime}{ }_{L L}$ are most likely due to some cantilever inhomogeneities or to non-ideal clamping, effects which are not yet taken into account in our model. Nevertheless, except for $G^{\prime}{ }_{L L}$, the cantilever-based shear modulus measurements seem to corroborate and extend the DWS reference measurements up to $100 \mathrm{kHz}$.

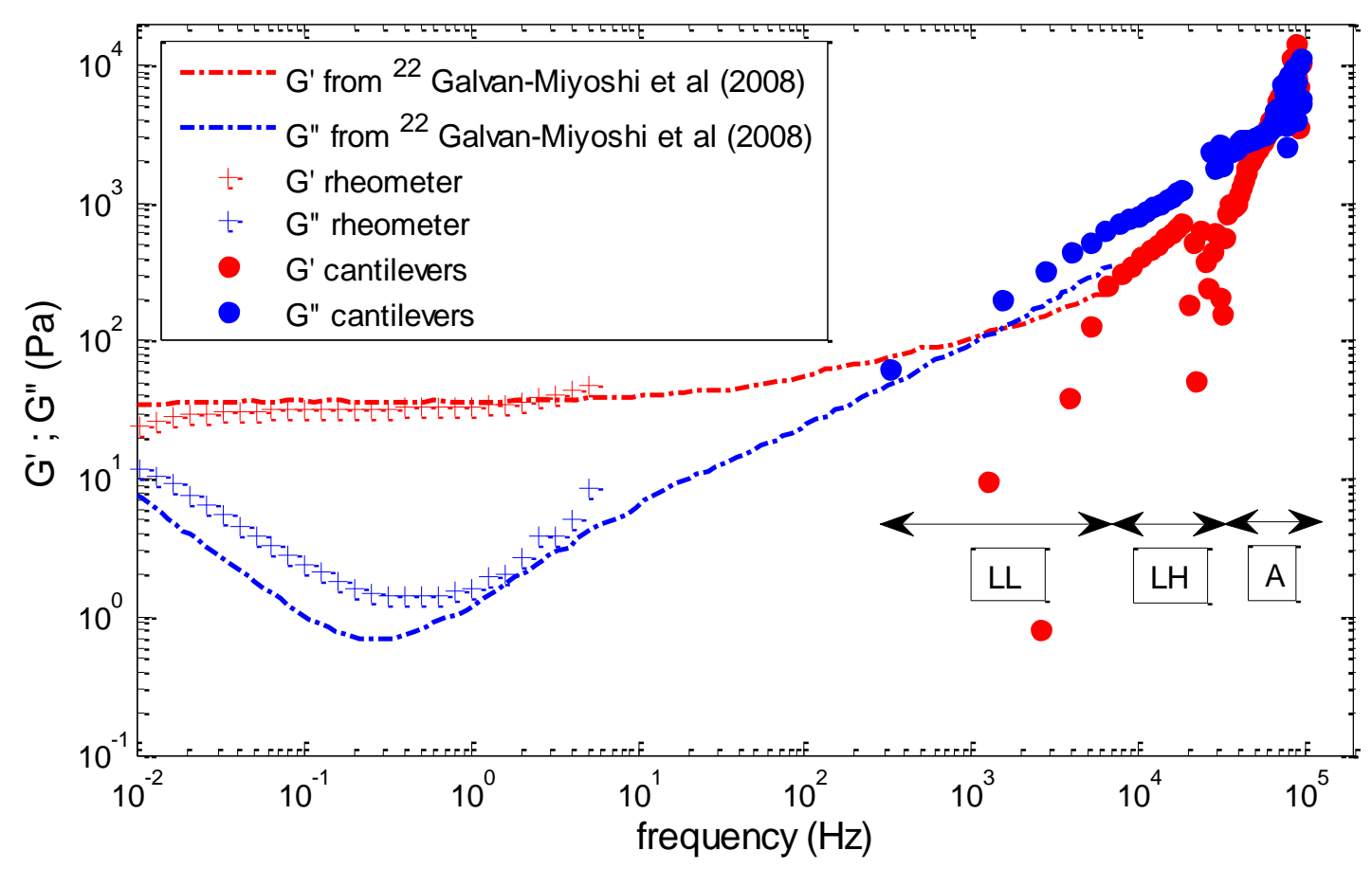

Fig. 11 - Rheogram of CTAB over a $\left[10^{-2}-10^{5}\right]$ frequency range measured with a classical cone-plate rheometer, with three different cantilever geometries using the inverse model (Eqs. 8-9 and 11) and reported from DWS measurements in the literature.

For the two concentrations of PAM (Fig. 12), very encouraging results are obtained for the viscous part $G$, , both in terms of its order of magnitude, qualitative trend and concentrationdependence. On the contrary, results for the elastic modulus $G$ ' are more bothersome. Below $5 \mathrm{kHz}$, these results are inconsistent, even though one could consider the elastic part to be much smaller than the viscous part. Above $5 \mathrm{kHz}, G$ ' exhibits a counter-intuitive concentration-dependent behavior: the elastic part seems to be decreasing with increasing concentration whereas the low- 
frequency behavior up to $100 \mathrm{~Hz}$ (e.g. measured in [25]) suggests the opposite. This surprising result is under investigation but at the moment we consider the obtained elastic value as inconsistent in this precise case due to a parametrical error, as will be discussed in the next section.

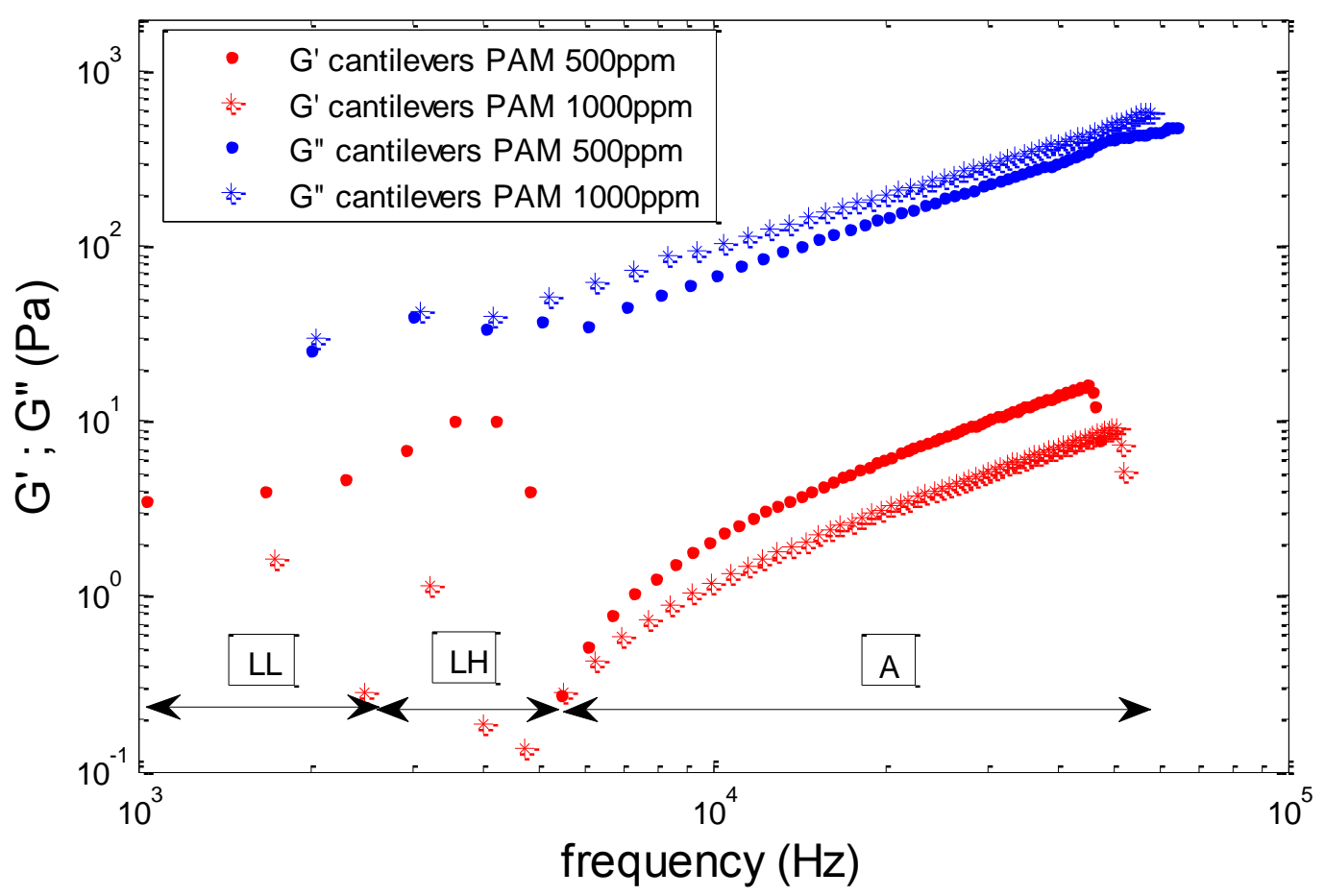

Fig. 12 - High-frequency rheogram of PAM (two concentrations) measured with three cantilever geometries (LL, LH and A) and spanning the frequency range $10^{3}$ $10^{5} \mathrm{~Hz}$ using the inverse model (Eqs. 8-9 and 11).

\section{Discussion}

In this paper we have demonstrated proof of concept for a new method for microrheological measurements but have also pointed out some limitations of the proposed method. The positive aspects are primarily related to the determination of $G$ ": an accurate measurement of the viscous part $G$ " is obtained showing good continuity between the results obtained with the different cantilevers. This would not be possible without improvements made to the modeling effort as documented herein. The limitations mainly concern the determination of $G^{\prime}$ : at present the method appears to yield valid results only for high values of $G$ ' and gives inconsistent results for smaller values (below $100 \mathrm{~Pa}$ ), thus warranting further physical verification an/or model improvement.

For small values of $G^{\prime}$ (that is, for Newtonian or near-Newtonian fluids), one part of the problem may stem from the linearization performed in solving the inverse problem, as shown in Fig. 4. The other source of problem, and perhaps the more significant, is related to experimental issues, since Figs. 9 and 10 show larger discrepancies for experimental $G$ ' values than for their simulated $G$ ' counterparts when comparisons are made to the expected results. For higher values of $G$ ' (that is, for non-Newtonian fluids), linearization yields no problem (see Fig. 5) but major issues continue to appear with experimental results (see Fig. 12).

It is worth mentioning that classical rheometers also have physical artifacts that lead to non-zero measured values for $G^{\prime}$ of Newtonian fluids of up to $10^{-2} \mathrm{~Pa}$ (see Fig. 10). In the case of microcantilevers using the proposed method this phenomenon still exists with increased anomalous behavior in $G$ ' being exhibited at higher frequency. The reason why $G$ ' is problematic whereas $G$ " 
is not requires further investigation. It could possibly be due to the very high sensitivity of the calculation to the system parameters and measurement data $\left(\omega, w(L, \omega), b, h, L, H_{0}, m_{L}\right)$ and the uncertainty in these quantities. In this case, a very small error during the calibration procedure, due to phase or amplitude of deflection measurement errors, could lead to a large error in property estimation. This is indicated by Fig. 6 and by similar figures that could be presented for the other parameters. For example, we have preliminary evidence that any experimental phase offset may be critical for $G$ ' determination. These problems could also be due to the proposed model itself. Should that be the case, the model could be generalized to take into account (i) internal losses in the beam, (ii) microscale temperature effects due to Joule heating in the gold path, (iii) effects of finite $b / h$ and $L / b$ ratios that are neglected in the present elementary beam theory model, and (iv) the inertial effects of the gold path at the free-end of our devices. Generalization of the model, however, would pose some technical challenges. For example, the inverse problem may no longer be solvable analytically (e.g., if the inertia of the gold is included) or addition system parameters may need to be incorporated (e.g., if the internal losses are modeled).

Another strategy for overcoming the above-mentioned obstacles can be used. In lieu of attempting to determine $G$ ' and $G$ " over a broad frequency range, the objective could be focused only on the properties at the eigenfrequency, in which case use can be made of a similar model [13]. Recently we achieved consistent measurement of $G$ ' and $G$ " for opaque viscoelastic fluids using microstructures [26]. Nevertheless the strategy we employed eliminates completely the advantage of the frequency-dependent analytical method developed in this work. A future analytical correction to the present model will be developed, based on some of the results provided by the model in the present paper. It will permit maintaining the generality of the analytical method developed here, which is valid for a wide range of cantilevers and fluids. Moreover, it will help to characterize accurately not only $G$ ”' but also $G$ ' as a function of frequency in a very general context.

To conclude, the viscous modulus obtained with the present method is consistent with linear trends for Newtonian fluids as well as with reference data from the literature for complex fluids; however, such consistency has not yet been achieved for the elastic modulus results. Further improvements to the model are currently being pursued, but in its present form it shows promise for measuring the viscoelastic tendencies of fluids at high frequencies.

\section{CONCLUSIONS AND FUTURE WORK}

Here we have demonstrated the potential for microcantilevers to be used to probe the highfrequency viscous modulus of small volumes of Newtonian and non-Newtonian fluids covering a specific frequency range from 1 to $100 \mathrm{kHz}$. The analytical method has been improved in order to eliminate the previous error ${ }^{3}$ of the shear modulus estimation method as a function of the complex free-end deflection. In addition some parameters have been identified as crucial for the accuracy of the experimental shear modulus determination; they have been optimized as much as possible given the present state of the model. Finally, high-frequency rheograms of four fluids, Newtonian and non-Newtonian, have been produced: they partially validate the presented inverse analytical model and prove the feasibility of a microcantilever-based microrheometer. Those two points have been carefully demonstrated, highlighting the actual advantages and limitations of this characterization method.

\footnotetext{
${ }^{3}$ As demonstrated in this paper, the previous error of the shear modulus estimation (developed in [14]) was due to the use of a simplified second order transfer function (Eq.10) to estimate the complex free-end deflection.
} 
One of the advantages of the proposed method is the very short computational time associated with the approach (on the order of seconds). Possible further improvements concerning the parameter determination methods and some modifications of the actual modeling have been noted. In particular, future improvements are needed to be able to measure accurately the elastic modulus $G$ '. Then, the integration of arrays of cantilevers, simultaneously performing measurements, is another direction for future work. Finally an electrical characterization of the cantilever deflection strain could possibly lead to an embedded microrheometer that could overcome practical issues associated with making measurements on opaque fluids.

\section{Acknowledgements}

The authors would like to thank Cédric Ayéla for the cantilever mask designs, and Liviu Nicu, Fabrice Mathieu and Daysuke Saya for the fabrication of the silicon cantilevers. They also would like to thank Yacine Amarouchène, Annie Colin and Hamid Kellay for useful discussions about microrheology. This work was partially supported by the CPER Pôle 4N Nanosciences en Aquitaine (GP-206-action 216/1) with the contribution of the Conseil Régional d'Aquitaine, the FEDER and the Ministry of Education and Research, by the French National Agency (MicRheo project $n^{\circ}$ ANR-08-NANO-004) and by the Conseil Régional d'Aquitaine ( $\left.\mathrm{n}^{\circ} 20091102001\right)$. 


\section{APPENDIX A: HYDRODYNAMIC FORCE FOR NEWTONIAN AND COMPLEX FLUIDS}

The hydrodynamic force per unit length, $F_{\text {hydro }}$, exerted by the fluid on the cantilever is composed of two terms (Eq. A1): one proportional to the microcantilever velocity (viscous coefficient $g_{l}$ depending on the radial frequency $\omega$ ) and one proportional to the microcantilever acceleration (inertial coefficient $g_{2}$ depending on the radial frequency $\omega$ ).

$$
F_{\text {hydro }}=-\omega^{2} g_{2}(\omega) w(x, \omega)-j \omega g_{1}(\omega) w(x, \omega)
$$

Furthermore, $g_{1}$ and $g_{2}$ can also be expressed as functions of both the real part $\Gamma_{r}$ and the imaginary part $\Gamma_{i}$ of the hydrodynamic function:

$$
\begin{aligned}
& g_{1}=\frac{\pi \rho_{\text {fluid }} b^{2}}{4} \omega \Gamma_{i}(\delta) \\
& g_{2}=\frac{\pi \rho_{\text {fluid }} b^{2}}{4} \Gamma_{r}(\delta)
\end{aligned}
$$

where $\delta=\sqrt{\frac{2 \eta}{\rho_{f} \omega}}=\frac{b}{\sqrt{2 R_{e}}}$ (with $R_{e}$ the Reynolds number as defined by Sader [16]). $a l^{20}$ :

The real and imaginary parts of the hydrodynamic function have been expressed by Maali et

$$
\Gamma_{r}=a_{1}+a_{2} \frac{\delta}{b}
$$

and

$$
\Gamma_{i}=b_{1} \frac{\delta}{b}+b_{2}\left(\frac{\delta}{b}\right)^{2}
$$

Considering the relation between the viscosity $\eta$ and the shear modulus $G$,

$$
G=j \omega \eta
$$

the expression of the hydrodynamic function becomes:

$$
\Gamma=a_{1}+\frac{a_{2}}{b \omega} \sqrt{\frac{-2 j G}{\rho_{f}}}+j\left[\frac{b_{1}}{b \omega} \sqrt{\frac{-2 j G}{\rho_{f}}}-\frac{2 b_{2} j G}{\rho_{f} \omega^{2} b^{2}}\right]
$$

If the fluid is complex (viscous and elastic): $G$ is complex $G=G^{\prime}+j G^{\prime \prime}$ and $\eta=\eta^{\prime}-j \eta^{\prime \prime}$ with $G^{\prime}>0, G^{\prime \prime}>0, \eta^{\prime}>0$ and $\eta^{\prime \prime}>0$

After a few calculations, Eq.A6 implies that

$$
\Gamma_{r}(\text { complex })=a_{1}+b_{2} \frac{2 G^{\prime}}{\rho_{f} \omega^{2} b^{2}}+\frac{1}{b \omega \sqrt{2 \rho_{f}}}\left[\begin{array}{c}
a_{2}+b_{1} \sqrt{\sqrt{G^{\prime 2}+G^{\prime 2}}+G^{\prime}} \\
+a_{2}-b_{1} \sqrt{\sqrt{G^{\prime 2}+G^{\prime 2}}-G^{\prime}}
\end{array}\right]
$$




$$
\Gamma_{i}(\text { complex })=\frac{2 b_{2} G^{\prime \prime}}{\rho_{f} \omega^{2} b^{2}}+\frac{1}{b \omega \sqrt{2 \rho_{f}}}\left[\begin{array}{c}
b_{1}-a_{2} \sqrt{\sqrt{G^{\prime 2}+G^{\prime \prime 2}}+G^{\prime}} \\
+a_{2}+b_{1} \sqrt{\sqrt{G^{\prime 2}+G^{\prime 2}}-G^{\prime}}
\end{array}\right]
$$

If the fluid is Newtonian (no elasticity): $G=j G^{\prime \prime}$ with $G^{\prime \prime}>0$ and $G^{\prime}=0$.

Therefore

$$
\begin{gathered}
\Gamma_{r}(\text { newtonian })=a_{1}+\frac{a_{2}}{b \omega} \sqrt{\frac{2 G^{\prime \prime}}{\rho_{f}}} \\
\Gamma_{i}(\text { newtonian })=\frac{b_{1}}{b \omega} \sqrt{\frac{2 G^{\prime \prime}}{\rho_{f}}}+\frac{2 b_{2} G^{\prime \prime}}{\rho_{f} \omega^{2} b^{2}}
\end{gathered}
$$

Note:

According to Sader [16] when $R_{e} \rightarrow \infty$ (e.g., when $G^{\prime \prime} \rightarrow 0$ ), for cylindrical and rectangular beams $\Gamma_{r}($ newtonian $)=1$ and $\Gamma_{i}($ newtonian $)=0$. Therefore:

$$
a_{1}=1
$$

The model of Maali et al [19] yields a very similar result for the value of $a_{1}$ as it was based on a fit of Sader's equations [16] ${ }^{\text {Erreur ! Signet non défini. }}\left(a_{1}=1.0553, a_{2}=3.7997, b_{1}=3.8018\right.$ and $b_{2}=2.7364$ are the "Maali's parameters").

If the fluid has only elasticity (no viscosity): $G=G^{\prime}, G^{\prime \prime}=0$. So:

$$
\begin{aligned}
\Gamma_{r}(\text { elasticity })= & a_{1}+b_{2} \frac{2 G^{\prime}}{\rho_{f} \omega^{2} b^{2}}+\frac{1}{b \omega \sqrt{2 \rho_{f}}}\left[a_{2}+b_{1} \sqrt{2 G^{\prime}}\right] \\
& \Gamma_{i}(\text { elasticity })=\frac{b_{1}-a_{2} \sqrt{G^{\prime}}}{b \omega \sqrt{\rho_{f}}}
\end{aligned}
$$

In this case because there is no viscosity and, thus, no damping effect; consequently $\Gamma_{i}=0$.

We can deduce that:

$$
b_{1}=a_{2}
$$

(The model of Maali et al [19] gives $b_{1} \approx a_{2}$ ).

\section{General case considering Eq. A11 and Eq. A14}

$$
\begin{gathered}
\Gamma_{r}(\text { complex })=1+b_{2} \frac{2 G^{\prime}}{\rho_{f} \omega^{2} b^{2}}+b_{1} \frac{2 \sqrt{\sqrt{G^{\prime 2}+G^{\prime \prime 2}}+G^{\prime}}}{b \omega \sqrt{2 \rho_{f}}} \\
\Gamma_{i}(\text { complex })=b_{2} \frac{2 G^{\prime \prime}}{\rho_{f} \omega^{2} b^{2}}+b_{1} \frac{2 \sqrt{\sqrt{G^{\prime 2}+G^{\prime 2}}-G^{\prime}}}{b \omega \sqrt{2 \rho_{f}}}
\end{gathered}
$$

Therefore, $g_{1}$ and $g_{2}$ are expressed as follow:

$$
g_{1}=\frac{\pi \rho_{f} b^{2} \omega}{4} \Gamma_{i}=b_{2} \frac{\pi}{2} \frac{G^{\prime \prime}}{\omega}+b_{1} \frac{\pi b \sqrt{\rho_{f}}}{2 \sqrt{2}} \sqrt{\sqrt{G^{\prime 2}+G^{\prime 2}}-G^{\prime}}
$$




$$
g_{2}=\frac{\pi \rho_{f} b^{2}}{4} \Gamma_{r}=\frac{\pi \rho_{f} b^{2}}{4}+b_{2} \frac{\pi G^{\prime}}{2 \omega^{2}}+b_{1} \frac{\pi b \sqrt{\rho_{f}}}{2 \omega \sqrt{2}} \sqrt{\sqrt{G^{\prime 2}+G^{\prime 2}}+G^{\prime}}
$$

These equations can be written as follow:

$$
\begin{gathered}
g_{1}=D G^{\prime \prime}+B \sqrt{\sqrt{G^{\prime 2}+G^{\prime 2}}-G^{\prime}} \\
g_{2} \omega=C+D G^{\prime}+B \sqrt{\sqrt{G^{\prime 2}+G^{\prime 2}}+G^{\prime}}
\end{gathered}
$$

where

$$
B=\frac{\pi b b_{1} \sqrt{\rho_{f}}}{2 \sqrt{2}} \quad C=\frac{\pi \rho_{f} b^{2}}{4} \omega \quad D=\frac{\pi b_{2}}{2 \omega}
$$

\section{APPENDIX B: COMPLEX SHEAR MODULUS AS FUNCTION OF THE TERMS $g_{1}$ AND} $\mathbf{g}_{2}$

The objective is to invert Eqs. A19-A20 to obtain $G^{\prime}$ and $G^{\prime \prime}$ as functions of $g_{1}$ and $g_{2}$.

Eqs. A19-A20 can be expressed as follows:

$$
\begin{gathered}
\left(\frac{g_{1}-D G^{\prime \prime}}{B}\right)^{2}=\sqrt{G^{\prime 2}+G^{\prime \prime 2}}-G^{\prime} \\
\left(\frac{g_{2} \omega-C-D G^{\prime}}{B}\right)^{2}=\sqrt{G^{\prime 2}+G^{\prime \prime 2}}+G^{\prime}
\end{gathered}
$$

By introducing the notation $K^{\prime \prime}=g_{1}-D G^{\prime \prime}$ and $K^{\prime}=g_{2} \omega-C-D G^{\prime}$, the difference of Eq. B1 and B2 gives

$$
K^{\prime \prime 2}-K^{\prime 2}=-2 B^{2} G^{\prime}=-\frac{2 B^{2}}{D}\left(g_{2} \omega-C-K^{\prime}\right)
$$

The product of Eq. B1 and Eq. B2 gives

$$
K^{\prime \prime 2} K^{\prime 2}=\frac{B^{4}}{D^{2}}\left(g_{1}-K^{\prime \prime}\right)^{2}
$$

From Eq. B4, it follows that

$$
K^{\prime 2}=\frac{B^{4}}{D^{2}}\left(\frac{g_{1}-K^{\prime \prime}}{K^{\prime \prime}}\right)^{2} \Rightarrow K^{\prime}=\frac{B^{2}}{D}\left(\frac{g_{1}-K^{\prime \prime}}{K^{\prime \prime}}\right) ; K^{\prime}>0
$$

Then using the definition of $K^{\prime}$ :

$$
G^{\prime}=\frac{g_{2} \omega-C-\frac{B^{2}}{D} \frac{g_{1}-K^{\prime \prime}}{K^{\prime \prime}}}{D}=\frac{g_{2} \omega-C-\frac{B^{2} G^{\prime \prime}}{g_{1}-D G^{\prime \prime}}}{D}
$$

Combining Eq. B5 and Eq. B3 yields 


$$
K^{\prime \prime 2}-\frac{B^{4}}{D^{2}}\left(\frac{g_{1}-K^{\prime \prime}}{K^{\prime \prime}}\right)^{2}=\frac{-2 B^{2}}{D}\left(g_{2} \omega-C-\frac{B^{2}}{D}\left(\frac{g_{1}-K^{\prime \prime}}{K^{\prime \prime}}\right)\right)
$$

This is a polynomial equation in $K$ " which can be written as

$$
K^{\prime \prime}+\left(\frac{B^{4}}{D^{2}}+\frac{2 B^{2}}{D} g_{2} \omega-C\right) K^{\prime \prime 2}-\frac{B^{4}}{D^{2}} g_{1}^{2}=0
$$

The positive solution of Eq. B8 is

$$
K^{\prime \prime 2}=\frac{-\frac{B^{4}}{D^{2}}-\frac{2 B^{2}}{D}\left(g_{2} \omega-C\right)+\sqrt{\left(\frac{B^{4}}{D^{2}}+\frac{2 B^{2}}{D}\left(g_{2} \omega-C\right)\right)^{2}+\frac{4 B^{4} g_{1}^{2}}{D^{2}}}}{2}
$$

Then using the $K$ " definition we obtain

$$
\begin{gathered}
G^{\prime \prime}=\frac{g_{1}}{D}-\frac{B}{D \sqrt{2 D}} \sqrt{\sqrt{\left(\frac{B^{2}}{D}+2 g_{2} \omega-C\right)^{2}+4 g_{1}^{2}}-\frac{B^{2}}{D}-2 g_{2} \omega-C} \\
G^{\prime}=\frac{1}{D}\left(g_{2} \omega-C-\frac{B^{2} G^{\prime \prime}}{g_{1}-D G^{\prime \prime}}\right)
\end{gathered}
$$

where

$$
B=\frac{\pi b_{1}}{2 \sqrt{2}} b \sqrt{\rho_{f}} \quad C=\frac{\pi}{4} \rho_{f} b^{2} \omega \quad D=\frac{\pi b_{2}}{2 \omega}
$$

\section{APPENDIX C: SOLUTIONS OF THE INVERSE PROBLEM}

\section{C.1 Solution of Eq. 11}

Three solutions $\left(Z_{1}, Z_{2}, Z_{3}\right)$ of Eq. 11 are obtained using a mathematical solver. Each analytical solution has been tested on simulated data and they correspond to the physical case (each solution corresponding to a different frequency range).This can be seen in Fig. C1 which represents the inertial term $\left(g_{2}\right)$ calculated using those three solutions.

Using the following notation, $n_{1}=3742200 ; n_{2}=2554051500 ; n_{3}=1260 ; n_{4}=311850 ; n_{5}=315$, Considering the definition of the terms, $T_{1}, T_{2}, U_{1}, U_{2}$ and $V_{1}$ :

$$
\begin{aligned}
& T_{1}=4 H+\frac{4}{3} \\
& T_{2}=\frac{H}{n_{3}}+\frac{1}{n_{4}}
\end{aligned}
$$




$$
\begin{gathered}
U_{1}=\frac{H}{n_{1}}+\frac{1}{n_{2}} \\
U_{2}=\frac{H}{3}+\frac{2}{n_{5}} \\
V_{1}=\left(\frac{T_{1}}{2 U_{1}}+\frac{T_{2}^{3}}{27 U_{1}^{3}}+\left(\left(\frac{T_{1}}{2 U_{1}}+\frac{T_{2}^{3}}{27 U_{1}^{3}}-\frac{U_{2} T_{2}}{6 U_{1}^{2}}\right)^{2}+\left(\frac{U_{2}}{3 U_{1}}-\frac{T_{2}^{2}}{9 U_{1}^{2}}\right)^{3}\right)^{\frac{1}{2}}-\frac{U_{2} T_{2}}{6 U_{1}^{2}}\right)^{\frac{1}{3}}
\end{gathered}
$$

The solutions $\left(Z_{1}, Z_{2}\right.$, and $\left.Z 3\right)$ of Eq. 11 are expressed as follows:

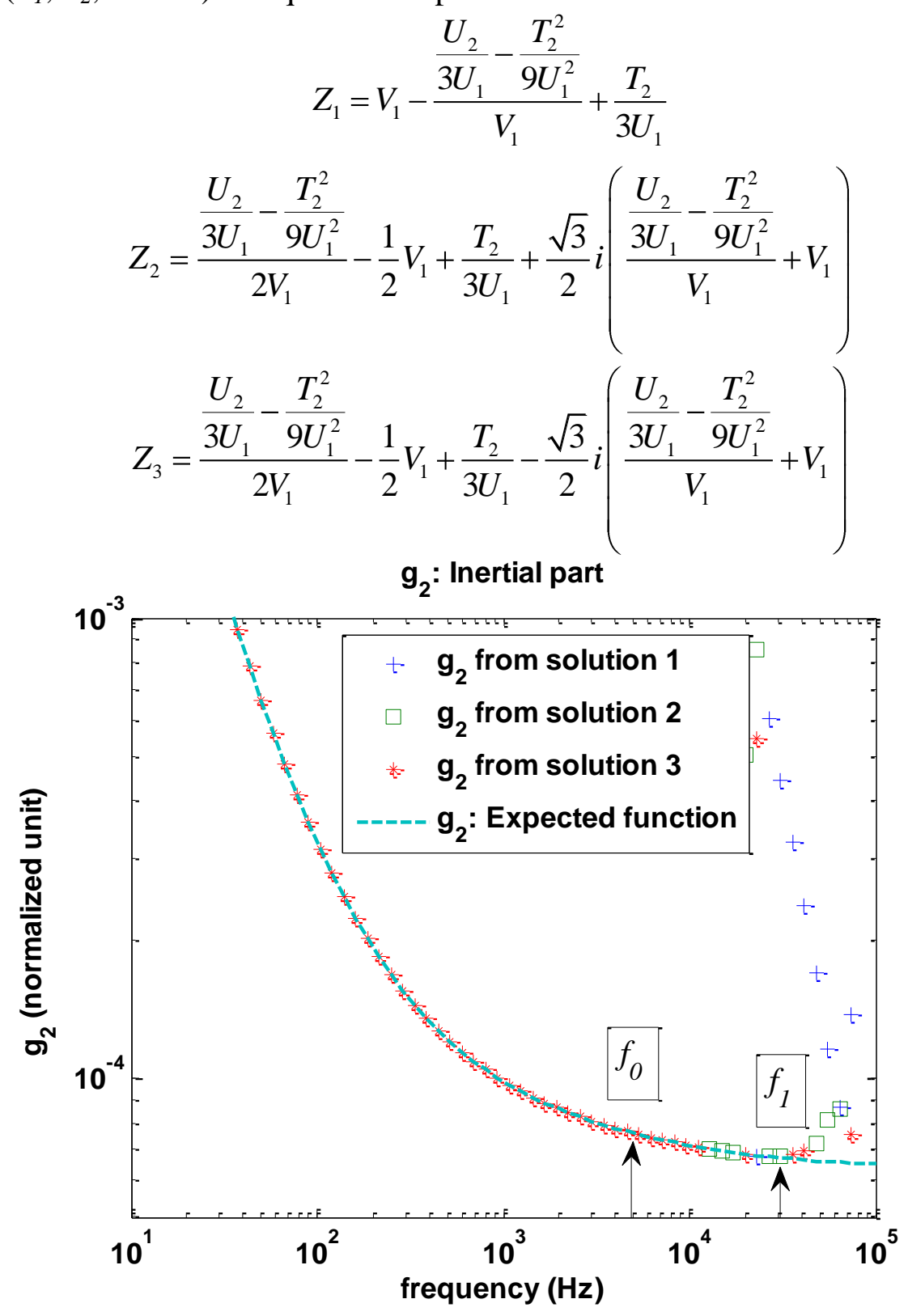

Fig. $\mathrm{C} 1$ - Inertial term $g_{2}$ calculated from the three inverse solutions $\left(Z_{1}:+, Z_{2}\right.$ : $\left.Z_{3}: *\right)$ of Eq.11 and the expected function which has been used to generate the simulation data. 


\section{C.2 Beyond Eq. 11}

Until now, Eq. 11 has been approximated by a ratio of $3^{\text {rd }}$-order polynomials, but it can be written exactly using an infinite sum since all the coefficients $k_{p}$ and $q_{p}$ can be calculated:

$$
H_{\text {Taylor }}(L, \omega)=\frac{\sum_{p=0}^{\infty} k_{p} Z_{i}^{p}}{\sum_{p=0}^{\infty} q_{p} Z_{i}^{p}}
$$

This equation can be written as

$$
\sum_{p=0}^{\infty}\left(k_{n}-q_{n} H\right) Z^{p}=0
$$

The three solutions $\left(Z_{1}, Z_{2}, Z_{3}\right)$ are the solutions of $(\mathrm{C} 10)$ if the sum is truncated at $p=3$ :

$$
\alpha_{0}+\alpha_{1} Z_{1|2| 3}+\alpha_{2} Z_{1|2| 3}^{2}+\alpha_{3} Z_{1|2| 3}^{3}=0
$$

with

$$
\alpha_{n}=\left(k_{n}-q_{n} H\right)
$$

The difference between the exact solutions of Eq. C10 and Eqs. C6-C8 can be included into a term called $\Delta$ which is added to each known solution $Z_{n}$ :

$$
\alpha_{0}+\alpha_{1}\left(Z_{1|2| 3}+\Delta_{1|2| 3}\right)+\alpha_{2}\left(Z_{1|2| 3}+\Delta_{1|2| 3}\right)^{2}+\alpha_{3}\left(Z_{1|2| 3}+\Delta_{1|2| 3}\right)^{3}+\ldots+\alpha_{p}\left(Z_{1|2| 3}+\Delta_{1|2| 3}\right)^{p}=0
$$

(C13) is a polynomial equation of $\Delta$ since both $\mathrm{Z}_{1|2| 3}$ and $\alpha_{\mathrm{i}}$ are known. Due to the properties of Taylor series development, the role of $\Delta$ is very small at low frequencies, but increases with frequency. Also, the weight of parameters $k_{i}$ and $q_{i}$ (and consequently $\alpha_{i}$ ) is decreasing while $i$ increases. Therefore, at low frequencies, the terms $\alpha_{i}$ and $\Delta^{j}$ can be neglected for the highest values of $i$ and $j$. For example, if (C13) is truncated at $i=6$ and $j=2$ (and taking (C11) into account):

$$
\begin{aligned}
& \alpha_{4} Z_{1|2| 3}{ }^{4}+\alpha_{5} Z_{1|2| 3}{ }^{5}+\alpha_{6} Z_{1|2| 3}{ }^{6}+\Delta_{1|2| 3} \alpha_{1}+2 \alpha_{2} Z_{1|2| 3}+3 \alpha_{3} Z_{1|2| 3}{ }^{2}+4 \alpha_{4} Z_{1|2| 3}{ }^{3}+5 \alpha_{5} Z_{1|2| 3}{ }^{4}+6 \alpha_{6} Z_{1|2| 3}{ }^{5} \\
& +\Delta_{1|2| 3}{ }^{2} \alpha_{2}+3 \alpha_{3} Z_{1|2| 3}+6 \alpha_{4} Z_{1|2| 3}{ }^{2}+10 \alpha_{5} Z_{1|2| 3}{ }^{3}+15 \alpha_{6} Z_{1|2| 3}{ }^{4}=0
\end{aligned}
$$

(C14)

For each of the three solutions $Z_{1}, Z_{2}$ and $Z_{3}$, Eq. C14 has two solutions, denoted by $\Delta_{1|2| 3}^{(a)}$ and $\Delta_{1|2| 3}^{(b)}$. Then, $Z_{1|2| 3}+\Delta_{1|2| 3}^{(a)}$ or $Z_{1|2| 3}+\Delta_{1|2| \beta}^{(b)}$ are more accurate solutions of Eq. 11, over a larger frequency range, than $Z_{1|2| 3}$. 


\section{REFERENCES}

[1] A. Agoston, F. Keplinger, B. Jakoby, Evaluation of a vibrating micromachined cantilever sensor for measuring the viscosity of complex organic liquids, Sensors and Actuators A 123-124, (2005), $82-86$

[2] G. F. Christopher, J. M. Yoo, N. Dagalakis, S. D. Hudson, K. B. Migler, Development of a MEMS based dynamic rheometer, Lab on Chip, 10 (2010), 2749-2757.

[3] M. L. Mather, Liquid viscoelasticity probed by a mesoscale piezoelectric bimorph cantilever, J. Rheol. 56(1), (2012). 99-112

[4] G. Fritz, W. Pechhold, N. Willenbacher, N. J. Wagner, Characterizing complex fluids with high frequency rheology using torsional resonators at multiple frequencies, J. Rheol. 47(2), (2003), 303319

[5] S.J. Martin, V.E. Granstaff, G.C. Frye, Characterization of a quartz crystal microbalance with simultaneous mass and liquid loading, Anal. Chem. 63 (1981), 2272-2281

[6] B. Jakoby, R. Beigelbeck, F. Keplinger, F. Lucklum, A. Niedermayer, E. Reichel, C. Riesch, T. Voglhuber-Brunnmaier, B. Weiss, Miniaturized sensors for the viscosity and density of liquids performance and issues, IEEE Trans. on Ultrason., Ferroelec., and Freq. Contr. 57 (2010), 111-120. [7] H. Freundlich, W. Seifriz, Ueber die elastizität von solen und gelen, Zeitschrift für Physikalische Chemie (1922), 104- 233.

[8] V. Breedveld, D.J. Pine, Microrheology as a tool for high-throughput screening, J. Mater. Sci., 38, (2003), 4461-4470.

[9] F.C. MacKintosh, C.F. Schmidt, Microrheology, Curr. Opin. Colloid Interface Sci. 4, (1999), 300-307.

[10] F. Ortega, H. Ritacco, R.G. Rubio, Interfacial microrheology: Particle tracking and related techniques, Curr. Opin. Colloid Interface Sci. 15, (2010), 237-245.

[11] P.Cicuta, , AM Donald, Microrheology: A Review of the Method and Applications, Soft Matter, 3, (2007), 1449-1455.

[12] S. Kirstein, M. Mertesdorf, M. Schönhoff, The influence of a viscous fluid on the vibration dynamics of scanning near-field optical microscopy fiber probes and atomic force microscopy cantilevers, Journal of Applied Physics, 84, (1998), 1782-1792.

[13] N. Belmiloud, I. Dufour, A. Colin, L. Nicu, Rheological behavior probed by vibrating microcantilevers, Applied Physics Letters 92, (2008), 041907,

[14] M. Youssry, E. Lemaire, B. Caillard, A. Colin, I. Dufour, A straightforward determination of fluid viscosity and density using microcantilevers: from experimental data to analytical expressions, Sensors and Actuators A, vol. 172, (2011), 40-46.

[15] I. Dufour, A. Maali, Y. Amarouchene, C. Ayela, B. Caillard, A. Darwiche, M. Guirardel, H. Kellay, E. Lemaire, F. Mathieu, C. Pellet, D. Saya, M. Youssry, L. Nicu, A. Colin, The Microcantilever: A Versatile Tool for Measuring the Rheological Properties of Complex Fluids, Journal of Sensors, Volume 2012 (2012), Article ID 719898, 9 pages.

[16] J. E. Sader, Frequency response of cantilever beams immersed in viscous fluids with applications to the atomic force microscope, J. Appl. Phys. 84, (1998), 64-76.

[17] A.J. Levine, T.C. Lubensky, Response function of a sphere in a viscoelastic two-fluid medium, Physical Review E, 63, nº4 (2001).

[18] F. Gittes, B. Schnurr, P.D. Olmsted, F.C. MacKintosh, C.F.Schmidt, Microscopic viscoelasicity: Shear moduli of soft materials determined from thermal fluctuations, Physical Review Letters, 79, (1997), 3286-3289. 
[19] A. Maali, C. Hurth, R. Boisgard, C. Jai, T. Cohen-Bouhacina, J.-P. Aimé, Hydrodynamic of oscillating atomic force microscopy cantilevers in viscous fluid, J. Appl. Phys., 97, (2005), 07490712.

[20] N. Belmiloud, I. Dufour, L. Nicu, A. Colin, J. Pistré, Vibrating microcantilever used as viscosimeter and microrheometer, Proceedings of the IEEE Sensors Conference, Daegu, Korea, (2006), pp. 753-756.

[21] Buchanan M, Atakhorrami M, Palierne J F, MacKintosh F C and Schmidt C F 2005 Highfrequency microrheology of wormlike micelles Phys. Rev. E 72011504

[22] www.laas.fr

[23] Mohamed Youssry, Etienne Lemaire, Benjamin Caillard, Annie Colin, Isabelle Dufour, Onchip characterization of the viscoelasticity of complex fluids using microcantilevers, Meas. Sci. Technol. 23 (2012), 125306 (10pp)

[24] J. Galvan-Miyoshi, J. Delgado, and R. Castillo, Diffusing wave spectroscopy in Maxwellian fluids, Eur. Phys. J. E 26, 369-377 (2008)

[25] Ahmad Darwiche, Microrheomètre sur puce pour l'étude de l'écoulement d'un liquide proche d'une surface solide, Thèse de l'Université Bordeaux1 (2012)

[26] Etienne Lemaire, Martin Heinisch, Benjamin Caillard, Bernhard Jakoby, Isabelle Dufour, Comparison and experimental validation of two potential resonant viscosity sensors in the kilohertz range, Meas. Sci. Technol. 24 084005, (2013) 